\title{
Phage Display Technology in Biomaterials Engineering: Progress and Opportunities for Applications in Regenerative Medicine
}

\author{
Ivone M. Martins, ${ }^{\dagger,+, \delta}$ Rui L. Reis, ${ }^{\dagger, \dagger}$ and Helena S. Azevedo ${ }^{*, \dagger,+, \|, \perp}$ \\ $\lceil 3$ B’s Research Group - Biomaterials, Biodegradables and Biomimetics, University of Minho, Headquarters of the European Institute \\ of Excellence on Tissue Engineering and Regenerative Medicine, AvePark, 4805-717 Barco, Guimarães, Portugal \\ ${ }^{\ddagger}$ ICVS/3B’s - PT Government Associate Laboratory, Braga/Guimarães, Portugal \\ ${ }^{\S} \mathrm{CEB}$ - Centre of Biological Engineering, University of Minho, 4710-057, Braga, Portugal \\ "School of Engineering \& Materials Science, Queen Mary University of London, London E1 4NS, United Kingdom \\ ${ }^{\perp}$ Institute of Bioengineering, Queen Mary University of London, London E1 4NS, United Kingdom
}

\section{Supporting Information}

ABSTRACT: The field of regenerative medicine has been gaining momentum steadily over the past few years. The emphasis in regenerative medicine is to use various in vitro and in vivo approaches that leverage the intrinsic healing mechanisms of the body to treat patients with disabling injuries and chronic diseases such as diabetes, osteoarthritis, and degenerative disorders of the cardiovascular and central nervous system. Phage display has been successfully employed to identify peptide ligands for a wide variety of targets, ranging from relatively small molecules (enzymes, cell receptors) to inorganic, organic, and biological (tissues) materials. Over the past two decades, phage display technology has advanced tremendously and has become a powerful tool in the most varied fields of research, including biotechnology, materials science, cell biology, pharmacology, and diagnostics. The growing interest in and success of phage display libraries is largely due to its incredible versatility and practical use. This review discusses the potential of phage display technology in biomaterials engineering for applications in regenerative medicine.

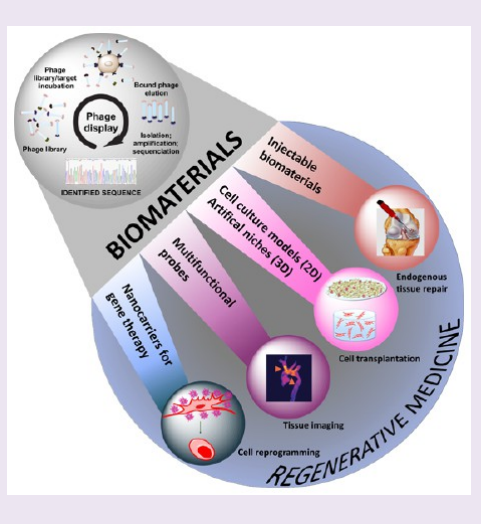

$\mathrm{R}$ egenerative medicine (RM) is a growing field of interdisciplinary research and clinical practices that has the potential of transforming the future of healthcare. It seeks to repair or replace malfunctioning cells, tissues, or organs caused by genetic disorders, chronic diseases, injuries, or aging. ${ }^{1}$ It employs a combination of in vitro/ex vivo and in vivo approaches that may involve transplantation of stem/progenitor, differentiated, or engineered cells, alone or incorporated in biomaterial scaffolds; tissue engineering; and delivery of therapeutic agents (e.g., cytokines, genes, small molecules) for reprogramming cell and tissue types or improving the function of the host environment. All RM strategies leverage the stimulation of the body's endogenous processes to develop and repair. Accordingly, stem cell research, gene therapy, biomaterials science, and molecular imaging play a central role in RM.

Phage display is a potent, high-throughput technology used for identifying peptide ligands for a given target. The technique applies a library of phage particles displaying an ample variety of peptides or proteins to identify those that bind to a certain target. The utility of this technology derives from phage biology, as phages can be genetically modified to express polypeptides on their surface while the gene encoding the polypeptide, found inside the viral particle, can be analyzed. Since its first establishment by Smith in $1985,{ }^{2}$ phage display has enabled the selection of a vast quantity of different peptides. Although mostly used to discover peptide ligands for mapping protein-protein interactions, and in the field of antibody engineering, phage display has evolved, now contributing to a variety of different areas of medicine and technology. Some of those areas that now benefit from the system are molecular and imaging diagnosis, ${ }^{3}$ peptide drug discovery, ${ }^{4}$ targeted drug and gene delivery, ${ }^{5}$ vaccine development, ${ }^{6}$ identification of new receptors and ligands, ${ }^{6}$ and nanomaterials. ${ }^{7,8}$ Several excellent reviews, covering different aspects of phages, including phage biology ${ }^{9}$ and the application of phage display in different areas, ${ }^{6,10-12}$ have been published.

Cell transplantation often requires prior expansion of the cells in vitro to obtain large numbers of functional cells for clinical applications. Thus, in vitro expansion is a critical step in cell-based therapies and typically involves the culture of cells on substrates coated with extracellular matrix (ECM) proteins. However, the batch-to-batch variability of ECM proteins presents limitations for both basic research and clinical applications of stem cells. Phage display technology provides a nonexpensive tool for the rapid and efficient screening of peptide ligands on cells or tissues, discriminating between subtle differences in cell surface phenotypes and between normal or diseased tissues. These peptide motifs can be incorporated into biomaterials to guide stem cell

Received: September 7, 2015

Accepted: September 23, 2016

Published: September 23, 2016 
proliferation and differentiation in vitro for subsequent transplantation, or to recruit local stem cells in vivo for stimulating tissue regeneration. When phage-derived peptides targeting specific cells or tissues are used, rather than the nonspecific peptide motifs routinely used in RM approaches, more controllable and effective regenerative therapies can be developed and tailored to individual patients, leading toward personalized medicine. In addition, phage display can provide novel peptides to investigate peptide-protein interactions that underlie disease mechanisms, which can be further used for designing potential therapeutic agents. Thus, peptide phage display is also a valuable tool in RM, and several therapeutic products generated by this technology are now commercially available, ${ }^{4}$ demonstrating its clinical potential. This review aims to recount the applications of phage display in biomaterials engineering and RM, highlighting the future of phage display in these fields.

Phage Display Screening - Biopanning. The most common screening method to identify peptide ligands for a given target by phage display (affinity selection) is known as biopanning. This procedure employs a phage display library that consist of a pool of individual clones $\left(\sim 10^{9}\right)$ bearing a different foreign DNA insert in the phage genome and presenting a different peptide on its surface. The external gene sequence is merged between genes encoding a signal peptide and a part of the capsid protein, which ensures that the a foreign peptide is expressed fused to the coat proteins. Molecules displayed on phage libraries are not restricted to peptides and antibodies. Random protein fragments, gene-fragment- or cDNA-encoded proteins, and mRNA phage display libraries have also been created, ${ }^{13,14}$ broadening the practical applications of the technology and demonstrating its versatility.

Typically, the process starts with library exposure to the target of interest to allow binding to the target under suitable conditions. The loosened phages are eliminated by washing, and the remaining bound phages are then eluted for subsequent bacterial infection and amplification. Viral multiplication occurs within the host cells, and thousands of newly formed phage particles are generated for additional selection rounds (usually $2-5$ rounds). After multiple panning rounds, enrichment of the target-binding phage is analyzed by phage titering and/or immunological assays. When sufficient enrichment is achieved, single phages are separated and sequenced to identify predominant binding motifs (consensus sequence). Selective clones may be obtained by examining the differences between unselected and selected libraries using DNA sequencing. ${ }^{15,16}$

Selection of ligands able to distinguish particular features in biological scenarios (cells and tissues) with good specificity and affinity is required for reliable diagnosis and effective regenerative therapies. Hence, potential targets in RM are signaling proteins (e.g., growth factors), cells, and tissues. These targets, however, have many potential binding sites, and nonspecific binding to abundant molecules (e.g., albumin) is also believed to affect the outcome of the panning experiments. Hamilton and co-workers ${ }^{17}$ described the application of phage display to select peptides that bind to bone morphogenetic protein 2 (BMP-2), which is implicated in osteoblast differentiation and stimulates bone formation. Using a recombinant form of human BMP-2 (rhBMP-2), they first biotinylated the protein for immobilization on streptavidin (SA)-coated microtiter plates. Biopanning was performed according to conventional protocols. After three cycles of screening, the populations of enriched phages were amplified in bacteria, and the binding to BMP-2 was detected by an enzyme linked immunosorbent assay (ELISA).
Many studies do not take into account the fact that the high density of receptors at the selection surface does not reproduce the density of receptors present on the native cell membrane. Thus, the binding affinity of the isolated peptide might be lower than the one observed when displayed on the phage. For example, Bastings et al. ${ }^{18}$ demonstrated, using SA as a model multivalent receptor, that receptor density and ligand valency are important parameters associated with binding strength. They showed that phage affinity for SA-coated surfaces declines significantly into the nanomolar scale when the surface density of $\mathrm{SA}$ is reduced. By displaying the peptide ligand in a multivalent arrangement (e.g., dimer, tetramer, or pentameric peptide wedge), they were able to increase (104-fold) the binding affinity as compared to the monovalent peptide. This study suggests that for effective targeting strategies, both the ligand presentation and the density of the corresponding receptor are fundamental aspects to consider. The ligand selection should be conducted on surfaces with physiologically representative receptor densities, and the peptides identified by phage display should be displayed in an appropriate form (e.g., dendrimer) when engineering biomaterials aiming for successful targeting. In addition, the cell surface contains proteins that can cluster or multimerize, and some of these organization patterns can be disturbed upon preparation of membrane proteins for selective screening. Indeed, the hydrophobic segments of membrane proteins direct them into a lipophilic environment, with such proteins naturally aggregating after separation from the membrane. Recombinant forms of these proteins have been widely used, but recombinant receptors are not always available. Hajduczki et al. ${ }^{19}$ proposed a new approach for the solubilization and presentation of membrane proteins, by proposing a different strategy for phage display selection. They prepared a phage library displaying caveolin-1 (monotopic membrane protein associated with detergent-insoluble rafts) variants generated by site-directed mutagenesis and used an antiselection process, consisting of binding to a hydrophobic resin, to eliminate aggregation-prone hydrophobic variants. The less hydrophobic variants were subsequently recovered and used in positive selection to isolate variants binding to the gp41 ectodomain (a known caveolin function). This additional positive panning ensured that the obtained variants were correctly folded. Thus, phage display screening on whole cells offers more chances to obtain peptides that bind to transmembrane proteins in their natural conformation. Panning on living cells can be done either on monolayers of attached cells or on cells in suspension. In these cases, the pool of target-binding phages needs to be diverse, but it can be reduced by performing a negative screening (preclearing) on "control" cells ${ }^{20}$ or a funnel screening protocol. Kiessling's group $^{21}$ used a screening approach based on the biopanning and rapid analysis for selective interactive ligands (BRASIL) method, previously developed by Giordano and co-workers, ${ }^{20}$ to isolate peptides binding to the surface of live stem cells. BRASIL is more specific (panning of cells in suspension) and faster than conventional methods that depend on washing or limiting dilution. Because living stem cells present many different receptors on their surfaces, and no appropriate "control cells" were available for a negative selection step, they further optimized the screening to narrow down the pool of cell-binding peptides. They incubated the cells (human embryonal carcinoma cell line) with a mixture (1:1) of a peptide-bearing (library) and peptide-free (wild type) phage and monitored phage titer at each panning and washing step. The phage members that remained bound to cells after all washing stages were then subjected to an additional cycle 


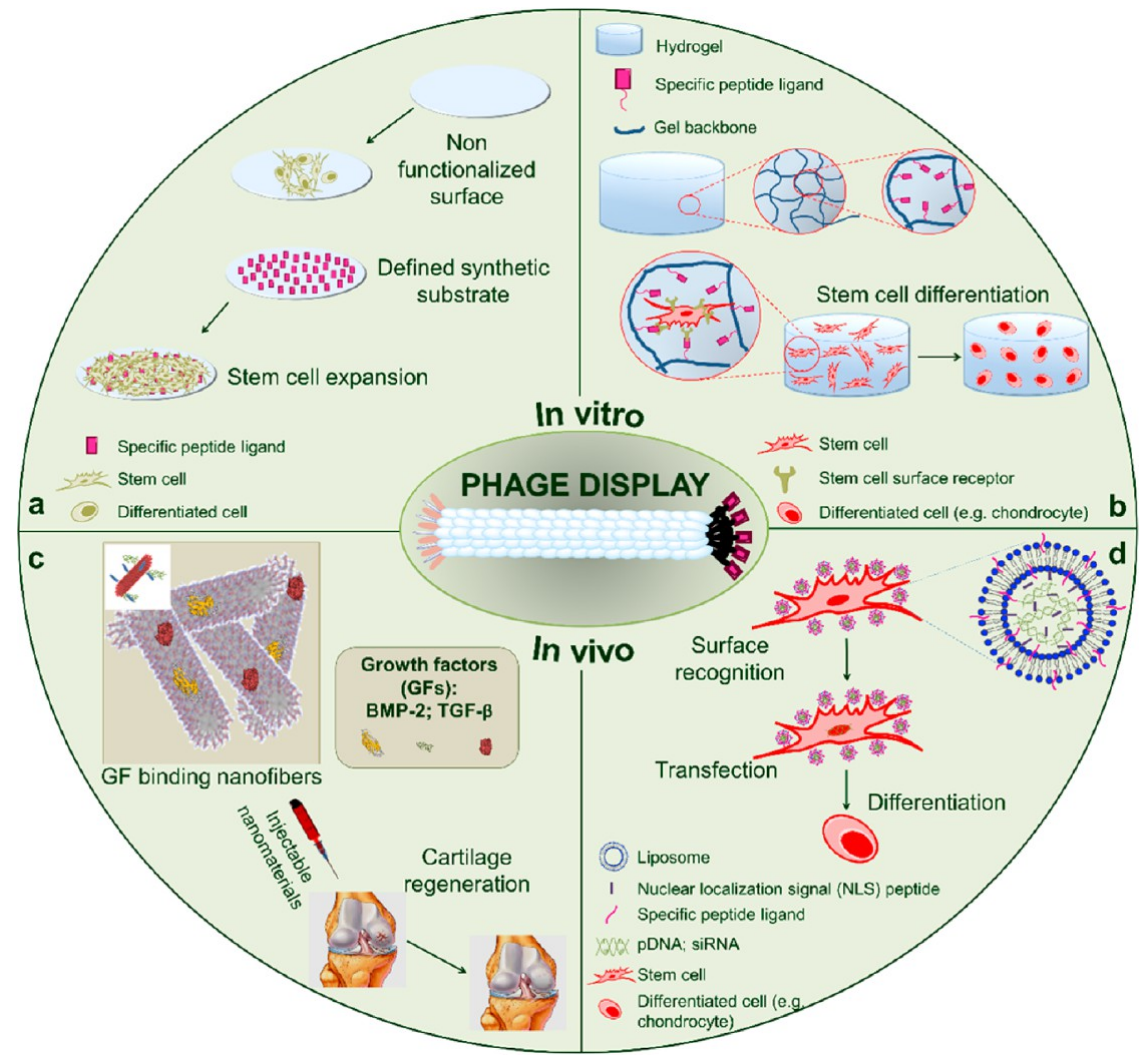

Figure 1. The use of phage display (center: M13 phage displaying a random peptide library on coat-protein 3 ) in biomaterials engineering and their applications in in vitro (a, b) and in vivo (c, d) RM approaches. (a) Functionalized synthetic substrates (2D surfaces) for stem cell expansion. (b) Functionalized hydrogels (3D environments) for recreating/manipulating stem cell niches. (c) Functionalized injectable biomaterials for sequestering GFs to promote endogenous tissue repair. (d) Functionalized nanocarriers for cell reprogramming (target delivery of genetic material to specific cells).

of incubation and washing, resulting in 50-fold enrichment of the peptide-containing phage. However, many nonspecific phages remained in the pool. Therefore, to select the cell-binding phage, they employed another screening step using ELISA. Seven cellbinding phage clones were isolated from the 370 clones tested.

Since the human body is made of around 1014 cells with distinct types and the surface changes with the state of the cell, the problem gains further complexity in vivo. Although most biopanning experiments are conducted in vitro, it is recognized that the in vivo conditions greatly influence the expression of cell surface molecules and that the phenotype of certain cells changes when they are cultured in vitro. Thus, the application of phage display selection in vivo circumvents these challenges and allows the identification of ligands capable of homing to specific tissues or organs. ${ }^{6,22-25}$ In order to achieve this process, phage libraries are injected intravenously into animals and allowed to circulate for a certain time. While nonspecific phages tend to be found throughout the whole organism, phages with selective binding will concentrate in certain tissues or organs. The bound phages are recovered and amplified, and their DNA is sequenced. Pasqualini and Ruoslahti ${ }^{24}$ were the first to report this approach, isolating peptides that home to renal and cerebral vascular endothelium in vivo. In vivo panning has also been applied to identify peptide ligands with potential applications in RM. For example, Nowakowski et al. ${ }^{26}$ were the first to conduct a phage display experiment involving the bone marrow and stem cells. They injected the phage display library into the tail vein of mice and, $10 \mathrm{~min}$ after injection, isolated the bone marrow to elute phages bound to bone marrow cells. The eluted phages were purified, amplified, and then reinjected. Five rounds of screening resulted in the identification of a peptide that preferentially homes to bone marrow, also binding to resident hematopoietic stem cells. The receptor of this peptide may be involved in bone marrow engraftment by recruiting hematopoietic stem cells.

One of the most laborious and costly steps (rate-limiting) in phage display screening is the DNA sequencing of the encoded displayed peptides. Next generation sequencing (NGS), such as Illumina deep sequencing technology, permits a high-throughput and cost-competitive phage display selection because it can characterize over $10^{7}$ reads in a single run, providing more complete coverage of the libraries. ${ }^{27}$ This is particularly useful for panning on RM targets such as cells, which present hundreds of different receptors. For example, Hoen and colleagues ${ }^{28}$ applied NGS to analyze the phage library at different stages of the selection process when panning on KS483 osteoblast cells, finding positive hits after one round of selection. Since its first application, NGS has been applied to enhance phage display protocols, ${ }^{28-31}$ allowing the isolation of phage binders after the first biopanning round, and the selection of less frequent but more specific peptides. As an example, a new method for selection against targets located on the cell surface, which are less abundant and cannot be purified, was implemented by the Lerner lab. ${ }^{32}$ This method allows for the identification of ligands for lessfit molecules with lower affinity. Having ligands with lower affinity can be useful for neutralizing the function/activity of the target through binding to different regions.

In the following sections, we describe applications of phagederived peptides in the functionalization of novel biomaterials with potential implications in RM strategies. 


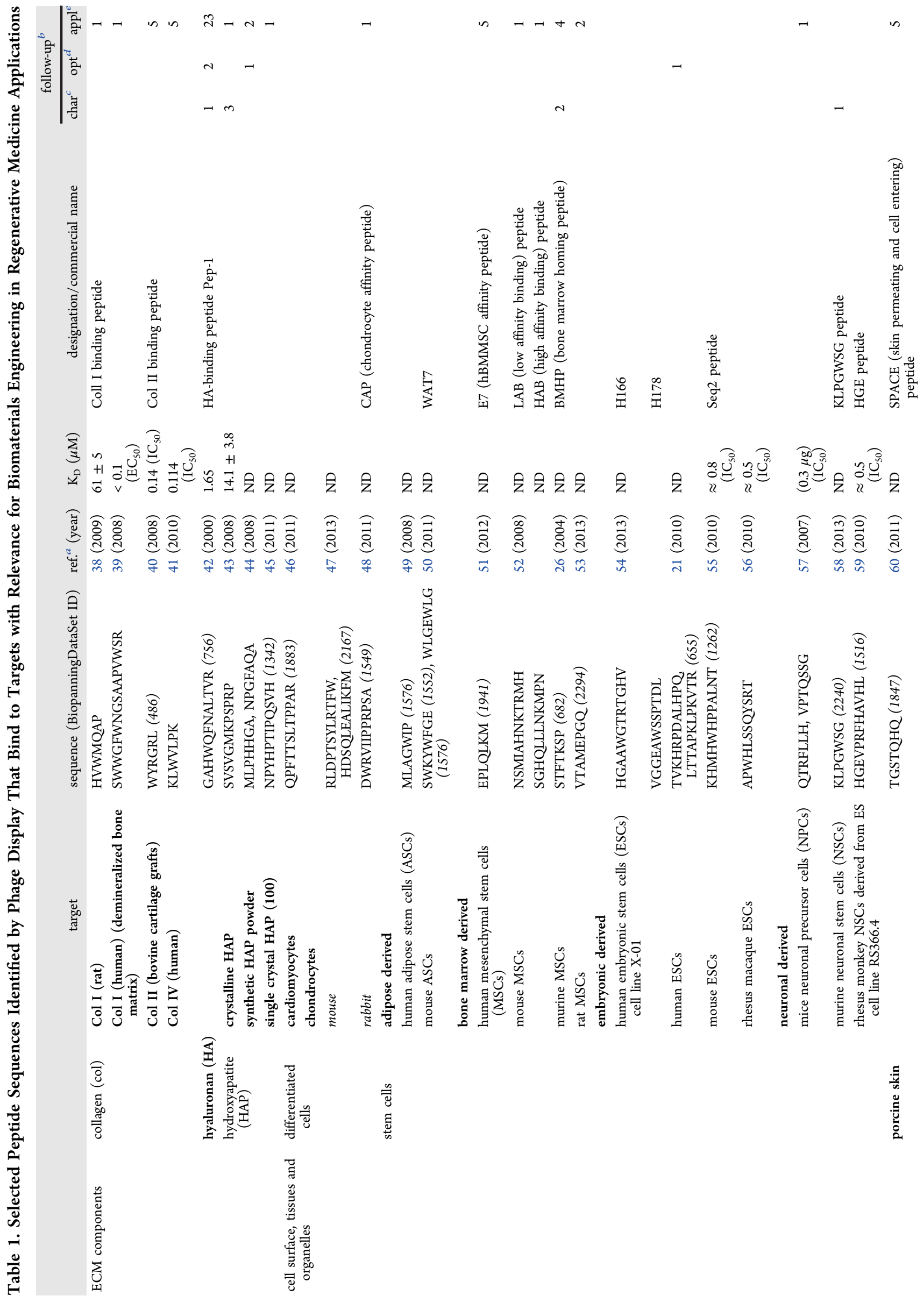




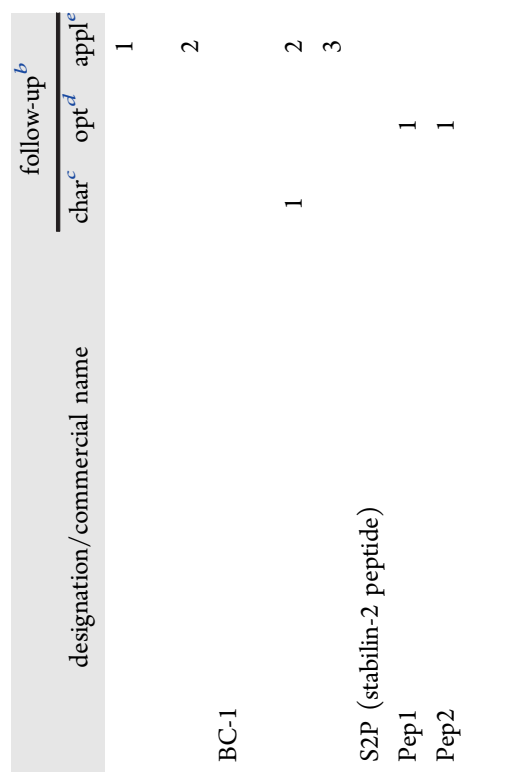

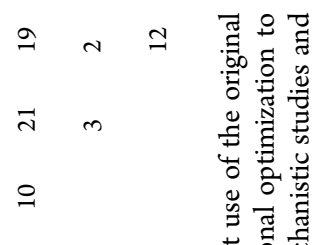

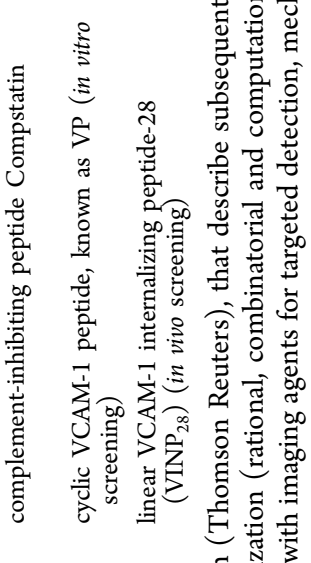

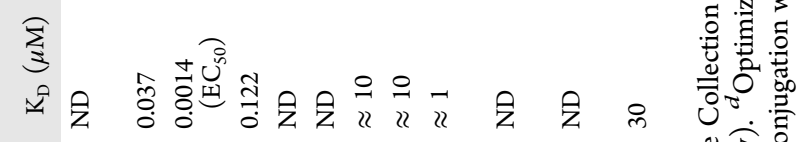

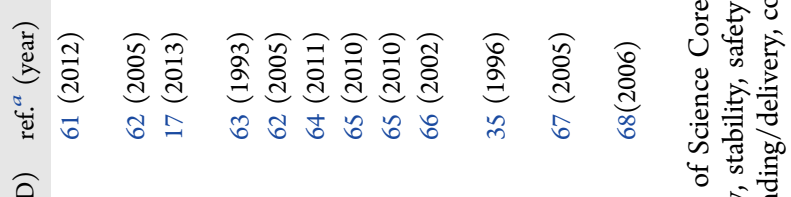

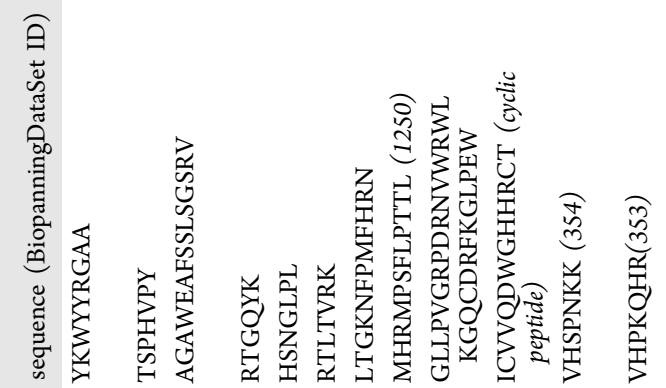

के 송

उ 穵

昰节

窎叟苛

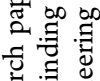

芯芯芯

㟧 옹

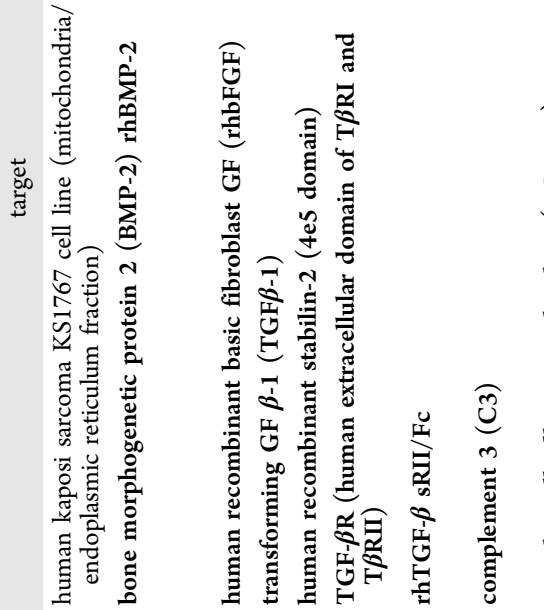

它 范

宓递

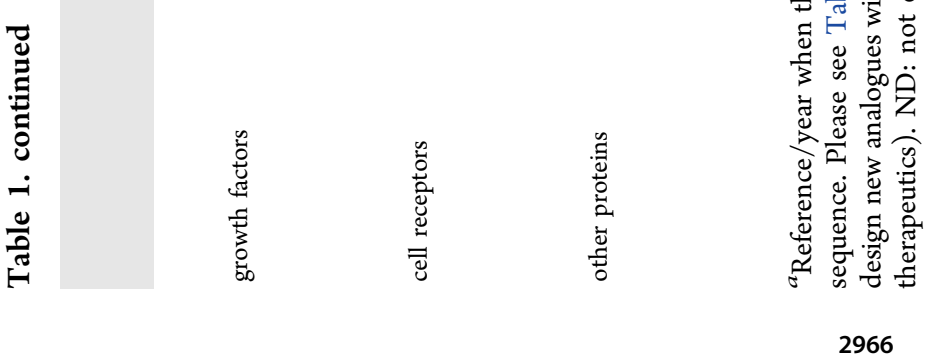


APPLICATIONS OF PHAGE DISPLAY IN BIOMATERIALS ENGINEERING FOR REGENERATIVE MEDICINE

The advances in phage display have greatly expanded the applications of the technology, creating new opportunities in biomaterials engineering and RM. Figure 1 shows examples of in vitro and in vivo $\mathrm{RM}$ strategies using different biomaterial systems functionalized with peptides identified by phage display. Phage-derived peptides, able to bind specifically to receptors on the surface of stem cells, can be used to functionalize synthetic scaffolds (2D surfaces and 3D hydrogels) for promoting stem cell expansion (Figure 1a) or differentiation (Figure 1b) in vitro. In vivo approaches include the incorporation of peptide ligands identified by phage display into injectable biomaterials (e.g., nanofibers), which can bind to growth factors (GFs) and promote endogenous tissue repair (Figure 1c), or into nanobiomaterials (e.g., liposomes, dendrimers), which can bind to receptors on the cell surface and deliver genetic material to target cells (gene therapy, Figure 1d).

Different databases (PepBank, Tree of Medicine, BDB) collating peptide sequences identified by phage display can be accessed free of charge, greatly contributing to data mining in this area. Currently, the biopanning data bank (BDB, at http:// immunet.cn/bdb/) formerly known as MimoDB ${ }^{33,34}$ contains 26813 peptide sequences, showing that the use of phage display has exploded over recent decades. However, many of the discovered sequences have not been further analyzed and validated in vitro or in vivo to assess specificity, selectivity, and stability, nor have they had their structure optimized to be clinically useful. To facilitate the search of sequences with utility for RM applications, we have compiled in Table 1 a selected list of peptide sequences that bind to RM targets (i.e., ECM components of tissues, GFs, surface of stem and differentiated cells). These peptide ligands can be used to specifically localize imaging probes or drugs to damaged tissues for diagnostic (immunohistochemistry, magnetic resonance imaging, positron emission tomography) and therapeutic (treating diseases) purposes; to deliver different biologics (e.g., GFs, oligonucleotides) to selected cell types for controlling their behavior (adhesion, survival, proliferation, migration, differentiation, angiogenesis); or to recruit local stem cells to sites of injury for stimulating tissue regeneration. The BiopanningDataSet ID was also included, when available, to facilitate the search in $\mathrm{BDB}$, as well as the equilibrium dissociation constant $\left(K_{\mathrm{D}}\right)$, which represents the binding affinity between the peptide ligand and the target, or the half inhibitory concentration $\left(\mathrm{IC}_{50}\right)$ from competition binding assays. In addition, a follow-up was performed to identify transformative publications that have identified breakthrough sequences, inspired new experiments based on the original sequence, and contributed to their application and clinical development, with potential impact on RM. To discriminate such contributions, quantitative data on the number of citations for each publication were first gathered, and from these, original articles were selected (excluding reviews, editorials, book chapters) to perform the analysis. While carrying out this evaluation, we must bear in mind that, typically, there is a $1-3$ year citation lag time for each article with impact, and certain sequences have been only recently published. Table 1 also summarizes the outcome of this analysis by identifying the number of publications that describe subsequent use of the original peptide sequence in diverse experimental studies, including in vitro and in vivo testing/characterization, and optimization/formulation toward their medical application. More details on this analysis can be found in Table S1 (Supporting Information). Many of the listed sequences have not resulted in any follow-up papers, mainly because they have been only recently discovered. One exception is compstatin, a cyclic tridecapeptide first identified by $\mathrm{Sahu}^{35}$ from phage-display libraries, which binds to complement component 3 (C3), a protein of the immune system. An excellent review on the "clinical trajectory" of compstatin was provided by Ricklin and Lambris. ${ }^{36}$ This peptide has contributed significantly to the understanding of compstatin-C3 interactions and the generation of new therapeutics (complement inhibitors). Compstatin, and in particular POT-4 (Table S1), which has been commercialized by Potentia Pharmaceuticals, Inc., has entered phase I clinical trials for the treatment of age-related macular degeneration and can be used for biomaterial functionalization to inhibit biomaterial-induced complement activation. ${ }^{37}$

In some cases, more than one sequence is identified for the same target. For example, when screening hydroxyapatite (HAP) materials, different powder particles, of various sizes and morphologies, used for the selection may explain the observed differences. Ideally, the selection should be performed using a material with specific morphology, crystallography, and stereochemistry. As described previously, several phage clones are selected in a first screening, and the relative affinity tends to increase with an increasing number of panning cycles. After this first screening, isolated clones are analyzed by ELISA, for example, for cross reactivity and binding ability. Sequences with varied affinities (high, intermediate, or low binding constants) can be obtained, thus allowing the engineering of binding affinity and specificity. Furthermore, by comparing the sequences obtained by phage display with the ones derived from natural proteins known to bind to the same targets, differences in the amino acid composition and sequence are also observed. For example, Mummert et al. ${ }^{42}$ found a 12-mer peptide that binds to hyaluronan (HA, Table 1) using phage display. This peptide sequence does not show significant $(>25 \%)$ similarity with the HA-binding motifs found in hyaladherins (CD44, RHAMM, or link protein $)^{69}$ nor does it show a consensus domain, known to be identical among these three HA-binding proteins. ${ }^{69}$ Nonetheless, the authors showed that the phage-identified peptide specifically binds to HA free in solution or bound to a substrate, and also to HA expressed on the cell surface, and that prevented the binding of HA to leukocytes and their attachment to HA coated surfaces. In addition, this sequence has been validated in numerous subsequent studies (Table S1).

Biomaterials Functionalized with Peptide Ligands Identified by Phage Display as in Vitro Cell Culture Models. The isolation and culture of human stem cells in vitro have largely contributed to important advances in fundamental cell biology and cell therapies, by understanding the molecular mechanisms involved in the processes by which stem cells undergo self-renewal or differentiation. ${ }^{70}$ Recent discoveries have shown novel mechanisms by which stem cell fate is regulated, ${ }^{71-73}$ but continued research in the molecular biology of stem cells will boost their application in RM. A challenge in cell-based therapies is to find adequate sources of stem cells and the need for maintaining them undifferentiated during in vitro expansion. Thus, there is an urgent need to develop precise and reproducible culturing systems able to control the behavior of human stem cells in vitro. In addition, for cell therapies in humans, an essential requirement is to avoid the exposure of stem cells to animal-derived components during culture. Advances in 


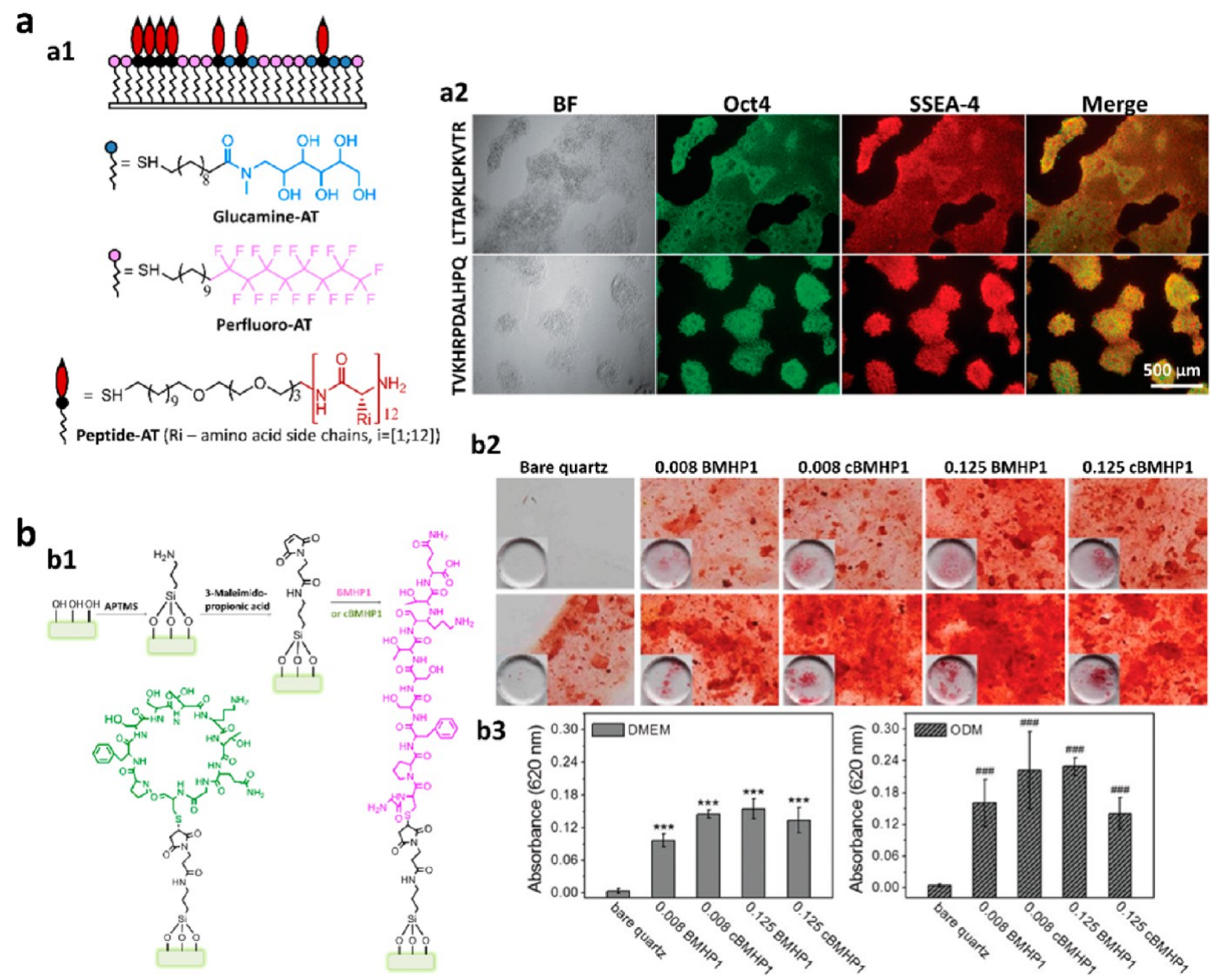

Figure 2. 2D biomaterials (surfaces) functionalized with peptide sequences identified by phage display for in vitro cell culture (proliferation and differentiation). (a) Synthetic surfaces of SAMs displaying phage-derived peptides that bind to the surface of human ESCs support their undifferentiated growth. (a1) SAMs composed of perfluorinated alkanethiols (ATs) containing different ratios of peptide-AT and glucamine-AT (nonadherent for cells) to obtain surface arrays of peptides with different densities. (a2) Human ESCs (hESCs) proliferating on peptide-AT SAMs maintain expression of markers of pluripotency (Oct4 and SSEA4). Adapted with permission from ref 21. Copyright 2010 American Chemical Society. (b) BMHP1 immobilized on 2D surfaces induce the osteogenic differentiation of MSCs. (b1) Linear and cyclic BMHP1 (cBMHP1) were conjugated to maleimidefunctionalized quartz substrates via a cysteine residue inserted in the N-terminus. (b2) Low-magnification (200X) inverted microscopy images of Alizarin Red S (forms a bright red complex with calcium) staining on MSCs cultured on bare and peptide-functionalized surfaces in DMEM (top panel) and ODM (bottom panel) for 7 days (insets show macroscopic images). (b3) Colorimetric quantification of calcium deposition by cells cultured in DMEM (left) and ODM (right). Adapted with permission from ref 75. Copyright 2015 The Royal Society of Chemistry.

biomaterials engineering have enabled the development of in vitro culture platforms for cell expansion, and also for the differentiation of stem cells into specific lineages. In vivo, cells receive signals from the ECM and other cells through specialized transmembrane proteins that bind to components of the ECM and receptors of neighboring cells. One potential approach to mimic these interactions and guide cell growth or differentiation in vitro is the use of biomaterials based on peptide sequences that bind to those cell receptors. Phage display has been applied to probe the surface of different stem cells, including adipose progenitor cells, ${ }^{49,50}$ bone marrow-derived mesenchymal stem cells (MSCs), ${ }^{26,51-53,74}$ and embryonic stem cells (ESCs) ${ }^{21,54-56}$ (Table 1). The latter are of special interest, considering their differentiation pluripotency. Peptide ligands for stem cell receptors can then be broadly used in cell culture and differentiation (immobilized on 2D surfaces or 3D hydrogels), namely, as probes for detection of specific stem cell populations during isolation procedures or to ascertain the role of proteins for stem cell differentiation.

2D Substrates for the Controlled Expansion (and/or Differentiation) of (Stem) Cells. Kiessling and collaborators ${ }^{21}$ used a peptide sequence identified by phage display (binding receptors on human embryonal carcinoma, Table 1) for culturing ESCs. Using self-assembled monolayers (SAMs) for displaying bound peptide ligands on a synthetic surface (Figure 2a1), they showed that these chemically defined functional substrates supported the undifferentiated proliferation of pluripotent cells (Figure 2a2). These cells are capable of replicating indefinitely and generate all human cell types, offering a useful resource for both research and RM. Thus, a surface with a well-defined composition could eliminate the problem associated with the use of animal-derived proteins, which suffer from batch-to-batch variability, and increase the predictability of culturing cells in vitro.

Similarly, MSCs cultured on peptide SAMs containing BMHP1 (Table 1, Figure 2b1) in maintenance medium (DMEM) or osteogenic differentiation medium (ODM) were able to produce higher amounts of calcium than when cultured on bare substrates (Figure 2b2,b3), demonstrating that BMHP1 can enhance cell mineralization. It was also shown that the amount of calcium deposition was dependent on the peptide density (Figure 2b3).

These studies showed the utility of the identified peptides for elucidating cell-matrix interactions in 2D. This information could be translated for the functionalization of $3 \mathrm{D}$ biomaterials to control cell behavior. 

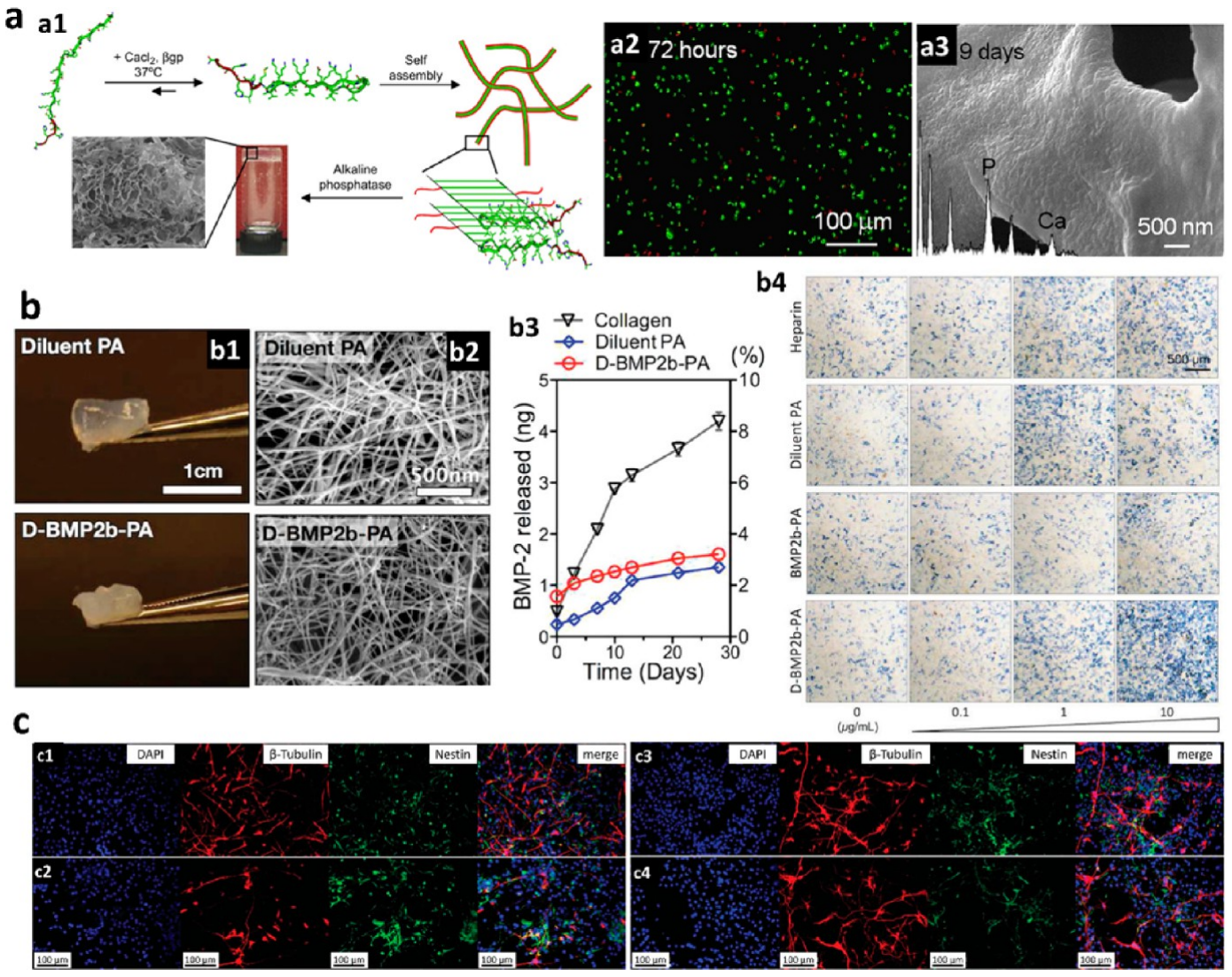

Figure 3. 3D biomaterials (hydrogels) functionalized with peptide sequences identified by phage display for in vitro cell culture (proliferation and differentiation). (a) Self-assembled peptide hydrogels functionalized with HAP-binding peptides for periodontal regeneration. (a1) Schematic illustration of the peptide self-assembly and hydrogel formation. (a2) Fluorescence microscopy image of a live/dead assay on cementoblast cells encapsulated within self-assembling peptide gels showing viable cells. (a3) Scanning electron microscopy (SEM) image of the peptide gel showing the deposition of calcium-phosphate mineral by cementoblasts, as confirmed by the corresponding EDXS (inset). Reprinted and adapted with permission from ref 78. Copyright 2010 Elsevier. (b) BMP-2-binding PA (BMP2b-PA) nanofibers induced osteoblast differentiation of a myoblast cell line (C2C12) in vitro. (b1) Photographs of PA-based gels (D-BMP2b-PA: obtained by mixing equal ratios of BMP2b-PA with diluent PA at the same concentration). (b2) SEM micrographs of PA gels showing the network of filamentous nanostructures. (b3) In vitro release of BMP-2 from PA gels, with or without BMP-2-binding PA, in comparison to collagen sponges preloaded with BMP-2 up to 28 days. (b4) Optical micrographs of C2C12 cells stained for the detection of ALP on day 3 of culture in a growth medium supplemented with treatment media containing $50 \mathrm{ng} \mathrm{mL} \mathrm{mof}^{-1}$ BMP-2 with heparin or PAs at different concentrations. Adapted from ref 87. Copyright 2014 Wiley-VCH Verlag GmbH \& Co. KGaA, Weinheim. (c) NSC differentiation on 3D peptide nanofiber gel scaffolds functionalized with bone marrow binding peptides, BMHPs. (c1-c4) Inverted fluorescence microscopy images of differentiating adult mouse NSCs cultured in vitro during 7 days on (c1) 1\% Matrigel (positive control), (c2) nonfunctionalized RADA16 peptide gel (negative control), (c3) RADA16-BMHP1, and (c4) RADA16-BMHP2, stained for cell nuclei (blue), $\beta$-tubulin ${ }^{+}$(red) for neurons, and nestin ${ }^{+}$(green) for neural progenitors (green). Adapted with permission from ref 83. Copyright 2006 Public Library of Science.

Growth factors (GFs) are involved in numerous cellular processes, such as cell growth, differentiation, and migration. TGF- $\beta$ is able to both positively and negatively affect inflammation and wound healing. Thus, several studies have been performed toward the identification of peptide ligands with selectivity and affinity to this GF and its receptors, aiming to understand their interactions and thus develop new strategies to tune their signaling capacity.

The regenerative potential of surfaces functionalized with TGF- $\beta 1$-binding peptide (Table 1 ) through azide-terminated SAMs and microcontact printing, and preloaded with TGF- $\beta 1$, was evaluated by culturing human articular chondrocytes (hACs) on the material. ${ }^{76}$ After 7 days of culture, more cells were observed on the peptide-functionalized surfaces than on the bare azide SAMs. Chondrocytes cultured in the absence of TGF- $\beta 1$ were found to be more extended, resembling the typical morphology of fibroblasts, while those cultured in the presence of TGF- $\beta 1$ exhibited a round polygonal shape, representative of chondrocyte phenotype. In addition, glycosaminoglycan (GAG) analysis revealed higher levels of GAGs in lysates of hACs cultured on peptide-functionalized surfaces with bound TGF- $\beta 1$ compared to hACs cultured on glass and with the GF free in the medium, and for cultures on peptide-functionalized surfaces without TGF- $\beta 1$.

Using phage display, two peptides that bind specifically to the TGF- $\beta$ receptor II (T $\beta$ RII; Table 1 ) have been identified. ${ }^{66}$ These peptides can mimic or block distinct functions of TGF- $\beta$ I and can be used to regulate these different functions, separately or in concert, which are important for vascular therapy. The group of Kiessling also used phage display to identify peptide ligands specific for TGF- $\beta$ receptors I and II ${ }^{65}$ (Table 1). They showed that the identified peptides bind to the receptors in a region different from the binding site used by TGF- $\beta$, thus not interfering with TGF- $\beta$ signaling. Therefore, these ligands can be applied as tools to examine the diverse cellular functions of TGF- $\beta$, as well as to promote the identification of potential therapeutics. Indeed, in later work, Kiessling and co-workers employed SAMs to present the 
a
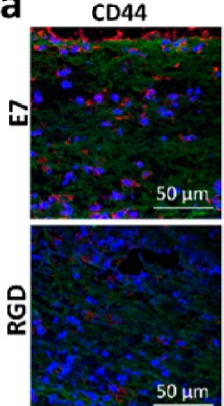

$0 \mu \mathrm{m}$

CD90

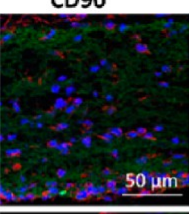

um:
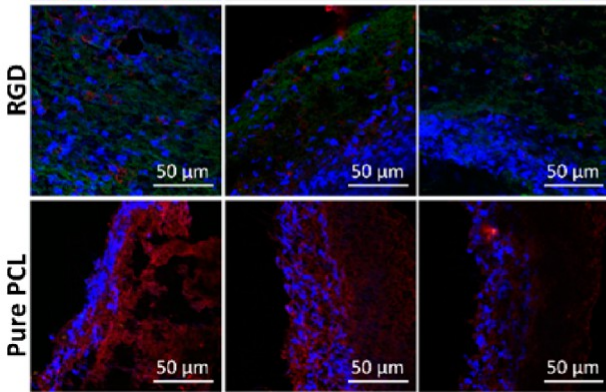

C c1

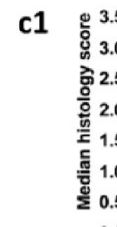

c2
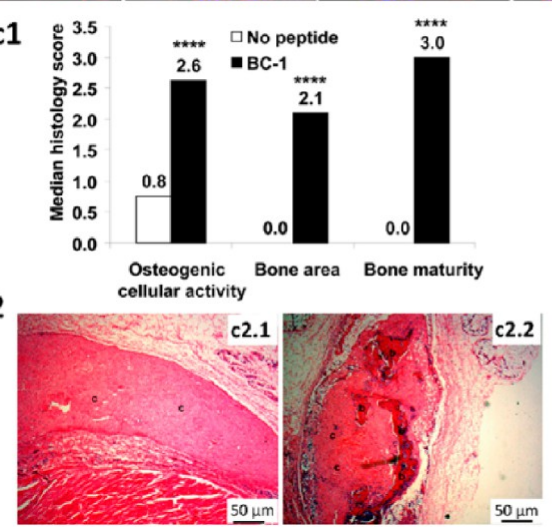

CD105

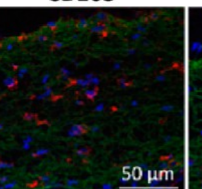

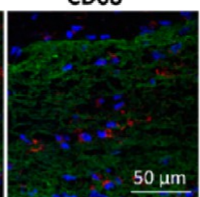

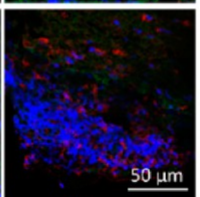

$\underline{50 \mu \mathrm{m}}$

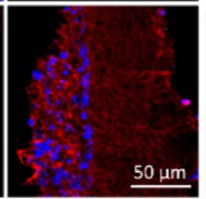

$50 \mu \mathrm{m}$

b3 b3.1

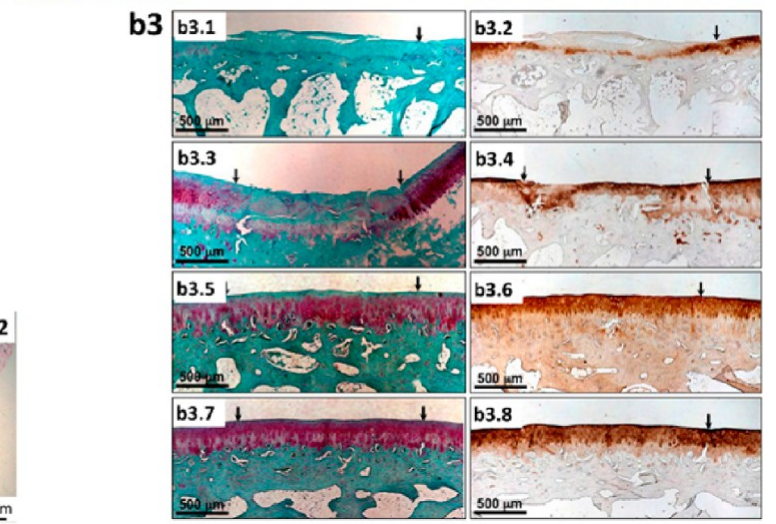

b b1
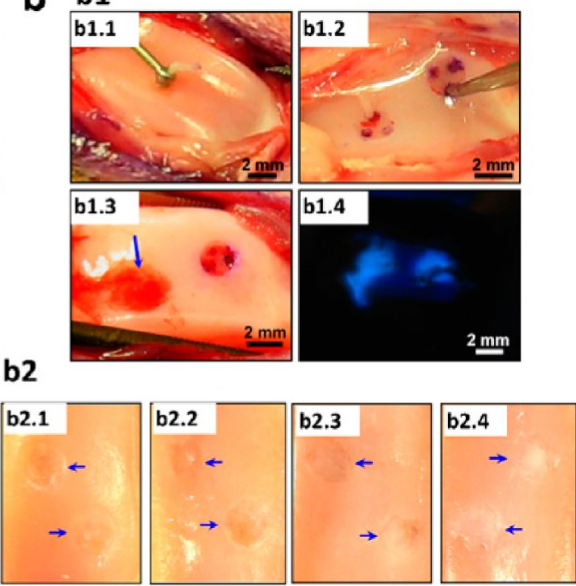

$+$

Figure 4. The application of phage-derived peptides for in vivo tissue regeneration. (a) In vivo stem cell homing by PCL electrospun meshes functionalized with E7 peptide with specific affinity for MSCs. (a1) Confocal microscopy images of peptide-conjugated PCL electrospun meshes harvested 7 days after implantation in full-thickness articular osteochondral defects in rats and stained by immunofluorescence for specific MSC (CD44, CD90, CD105) and inflammatory (CD68) markers (green: FITC, blue: Hoeschst33258, red: CD44/CD90/CD105/CD68 counterstained with Cy3 \& Cy5). Reprinted and adapted with permission from ref 51. Copyright 2012 Elsevier. (b) PA molecules displaying binding epitopes for TGF $\beta$ - 1 support the regeneration of full thickness chondral defects in articular cartilage. (b1) Surgical and implantation procedure showing (b1.1) full thickness articular cartilage defects made with a microcuret in rabbit trochlea; (b1.2) microfracture holes through the subchondral bone using a microawl to promote bleeding into the defect; (b1.3) injected PA gel in the defect (arrow); (b1.4) PA gel labeled with a fluorescent dye contained within the cartilage defects after injection. (b2) Images of articular cartilage defects after 12 weeks of implantation with (b2.1) $100 \mathrm{ng} / \mathrm{mL}$ TGF- $\beta 1$, (b2.2) filler PA + 100TGF, (b2.3) 10\%TGFBPA + 100TGF, (b2.4) 10\%TGFBPA alone. (b3) Microscopy images of tissue sections of articular cartilage defects obtained from histological and immunohistochemical analysis and stained for GAGs using safranin-O (left panel) and type II collagen (right panel) 12 weeks after treatment with (b3.1, b3.2) $100 \mathrm{ng} / \mathrm{mL}$ TGF- $\beta 1$, (b3.3, b3.4) filler PA + 100TGF, (b3.5, b3.6) 10\%TGFBPA + 100TGF, (b3.7, b3.8) 10\% TGFBPA alone. Reprinted with permission from ref 89. Copyright 2010 United States National Academy of Sciences. (c) An injectable collagen gel containing a bifunctional peptide (BC-1) with affinity for collagen and BMP-2 enhances retention of BMP-2 and increases ectopic bone formation; (c1) osteogenic cellular activity, bone area, and bone maturity scored from H\&E slides (c2) and by two observers; (c2) histology images (H\&E staining) from the rat ectopic model; (c2.1) $2 \mu \mathrm{g}$ BMP-2 in 1.5\% collagen gel; (c2.2) $2 \mu \mathrm{g}$ BMP-2 with 50-fold molar excess of BC-1 in 1.5\% collagen gel (b, bone zones; c, collagen zones; cells are stained blue). Adapted with permission from ref 17. Copyright 2010 Public Library of Science.

previously identified peptide sequences, which interact with both TGF- $\beta$ receptors (T $\beta$ RI-ED and T $\beta$ RII-ED), to provide localized and defined spatial signaling to cells. ${ }^{77}$ As a proof of concept, they used NMuMG mouse mammary gland cells to investigate the attachment of cells expressing TGF- $\beta$ receptors to the surfaces displaying the $\mathrm{T} \beta \mathrm{R}$-binding peptides. The cells bound to SAMs presenting either peptide (even at low peptide densities, 4\%), but not to the bare surfaces. They further demonstrated that the functionalized surfaces activated Smad2/3 nuclear translocation (a hallmark of TGF- $\beta$ signaling).

These studies demonstrate the power of phage display to generate peptides that can be used to regulate signaling pathways that lead to specific cell outcomes. Strategies to control the beneficial roles of signaling pathways would be valuable for applications in RM.
3D Hydrogels for Recreating Specific (Stem) Cell or Tissue Environments. Molecular design of biomaterials offers the opportunity to incorporate peptide ligands that can be recognized by specific cell receptors or enzymes, and to control their spatial and temporal availability for triggering regenerative events. The incorporation of peptide sequences identified by phage display into molecularly designed biomaterials, and in particular, self-assembling peptide biomaterials, has been reported by several groups.

Being the major inorganic constituent of bone and teeth, HAP has received great attention as a target for phage display experiments. For example, the Sarikaya group identified a HAP-binding peptide (Table 1 ), ${ }^{44}$ which was then incorporated into a peptide hydrogel capable of directing the mineralization of $\mathrm{HAP}^{78}$ (Figure 3a). Other studies describing peptides with preferential adsorption to HAP-based materials 
have also been reported ${ }^{45,79,80}$ (Table 1). Peptides with binding affinity to different apatite substrates can be used to direct the growth of HAP on biomaterials surfaces, ${ }^{81}$ or to improve the functional properties of HAP-based scaffolds, and thus enhance bone regeneration.

The Stupp laboratory designed self-assembling peptides, known as peptide amphiphiles (PAs), for RM applications. Of particular interest for the purpose of this review are PAs designed to bind GFs using peptide sequences derived by phage display. They first reported a novel class of reverse PAs to enable the creation of peptide assemblies with a free $\mathrm{N}$-terminus, which were not possible to obtain through the original design. They were able to apply this new methodology to synthesize PAs incorporating peptide sequences, derived via phage display and requiring a free $\mathrm{N}$-terminus, that bind GFs, BMP-2, and TGF- $\beta$ (Table 1), ${ }^{62}$ all involved in stem cell differentiation. These PA molecules are known to form nanofibers by selfassembly (Figure $3 \mathrm{~b} 2$ ), which at sufficiently high concentration is accompanied by gelation (Figure $3 \mathrm{~b} 1$ ). By permitting the direct binding of the GF to the PA nanofiber, gels containing BMP-2 bound to PA allowed prolonged GF retention (Figure 3b3). When in solution, the BMP-2-bound PA nanofibers induced the differentiation of $\mathrm{C} 2 \mathrm{C} 12$ premyoblast cells into osteoblasts, as detected by an increased number of alkaline phosphatase (ALP, marker for osteoblast differentiation)-positive cells (Figure 3b4).

The BMHP motif was used by Gelain and co-workers ${ }^{58,82-86}$ to functionalize self-assembling peptide scaffolds for culturing and controlling neural stem cell (NSC) behavior for nervous tissue regeneration. In their pioneering work, ${ }^{83}$ they used BMHP-functionalized self-assembling peptide hydrogel scaffolds for 3D culture of adult mouse NSCs. In differentiation assays, the scaffolds containing BMPH motifs (BMHP1 and BMHP2, Figure $3 \mathrm{c} 3, \mathrm{c} 4)$ showed $\beta$-tubulin ${ }^{+}$and nestin ${ }^{+}$cells comparable with those cultured on Matrigel (Figure $3 \mathrm{c} 1$ ). $\beta$-Tubulin ${ }^{+}$cells on $\mathrm{BMPH}$ scaffolds also showed increased branching when compared with nonfunctionalized scaffolds (Figure 3c2). These results demonstrate the ability of these synthetic peptide nanofiber scaffolds to be functionalized with different peptide motifs for the controlled 3D culture of diverse cell types.

Chemically cross-linked hydrogels, such as those composed of poly(ethylene glycol) (PEG), have been functionalized with different peptide ligands to culture cells in 3D. The Anseth group ${ }^{88}$ functionalized PEG hydrogels with a basic fibroblast growth factor (bFGF)-binding peptide, previously selected by phage display, ${ }^{63}$ to control GF retention and sustained release for applications in GF-induced regeneration. To enhance the affinity of the bound ligand for diffusible proteins (e.g., GFs) within the hydrogel, they synthesized trifunctional peptides and examined how the molecular structure of the affinity peptides affected their accessibility and binding using Forster resonance energy transfer. They then showed that the optimal functionalization strategy allowed the sustained release of bioactive bFGF able to induce the in vitro differentiation of the PC12 pheochromocytoma cell line. This affinity hydrogel could be manipulated for GF retention, or delivery, and be easily applied in distinct RM approaches, such as controlled stem cell differentiation in vitro or GF-mediated wound healing in vivo.

Implantable Biomaterials Functionalized with Peptide Ligands Identified by Phage Display for Promoting Endogenous Tissue Repair in Vivo. Tissue regeneration in vivo can be enhanced by promoting the recruitment and localization of stem cells into injured tissues and/or stimulating the function and differentiation of local stem cells. This can be achieved by manipulating the properties of the niche (e.g., promote angiogenesis or denovo niches) or through the delivery of active molecular regulators, directly or combined with a biomaterial, or by using biomaterial sequestering GFs.

Stem Cell Homing. Using polycaprolactone (PCL, an FDAapproved polymer for medical applications) electrospun meshes conjugated with phage-derived peptide (E7, Table 1) with affinity for human-bone-marrow-derived MSCs (hBMMSCs), Shao and co-workers showed improved recruitment of MSCs in vivo after implantation in full-thickness articular osteochondral defects in a rat knee. ${ }^{51}$ Immunofluorescence staining of harvested implants (Figure 4a) showed that cells on the E7-conjugated PCL meshes were mostly positive for CD44, CD90, and CD105 (MSCs surface markers), indicating that the functionalized scaffold could selectively recruit and retain MSC-like cells from the bone marrow. In contrast, cells in the PCL meshes conjugated with the cell adhesive peptide RGD (with no cell specificity, control) showed lower levels of MSCs surface markers. CD68 staining was also used to assess the inflammatory response. Results from immunofluorescence staining showed that there was a high proportion of inflammatory cells in the RGD-conjugated PCL meshes, while $\mathrm{CD}^{+} 8^{+}$cells were scarce in the E7-conjugated scaffold, suggesting only a minor inflammatory response. For the bare PCL meshes, only few cells could be detected into the mesh, due to the unfavorable cell adhesion properties of PCL. The E7 peptide could be further applied for functionalizing other biomaterials for effective MSC-homing in stem-cell-based tissue repair strategies.

Controlled Release of Growth Factors (GFs). TGF- $\beta 1$ is known to play a critical role in the development, growth, maintenance, and repair of articular cartilage. To this end, the Stupp group designed self-assembled peptide nanofibers for cartilage regeneration, using a peptide sequence derived by phage display with binding affinity for TGF- $\beta 1$ (Table 1$).{ }^{89}$ When implanted into a full-thickness chondral defect in a rabbit model (Figure 4b1), these self-assembled gels were shown to support the regeneration of articular cartilage (Figure 4b2,b3) with or without the supplementation of TGF- $\beta 1$, as detected by formation of hyaline-like tissue (GAGs and collagen II staining, Figure 4b3) within the defect space.

To promote bone healing, Hamilton et al. ${ }^{17}$ developed a novel strategy to deliver osteogenic GFs, such as BMP-2, in a collagen biomaterial carrier. This carrier contained a bifunctional peptide (BC-1) that displayed BMP-2 and collagen-binding domains, both identified by phage display (Table 1 ). This strategy allowed the simultaneous binding of GFs directly to an implantable biomaterial, without the requirement for any conjugation steps, thus allowing the controlled delivery of GFs at the implantation site. To test the ability of this system to promote bone formation in vivo, the authors injected BMP-2 within a collagen gel, containing or lacking $\mathrm{BC}-1$, into the subcutaneous regions of Sprague-Dawley rats (rat ectopic bone formation model). Histological analysis and evaluation of the explants showed significantly higher osteogenic cellular activity, bone area formed, and bone maturity in the presence of BC-1 (Figure 4c1,c2).

Degenerative joint diseases, such as osteoarthritis (OA), are characterized by progressive cartilage matrix degradation. Targeted therapies for damaged articular cartilage can offer the opportunity for localization of bioactive proteins, such as GFs, that can enhance the synthesis of a more hyaline-type cartilage. Toward this challenge, Hubbell's group described the synthesis and functionalization of nanoparticles for the release and retention of drugs inside articular cartilage through the identification 
a
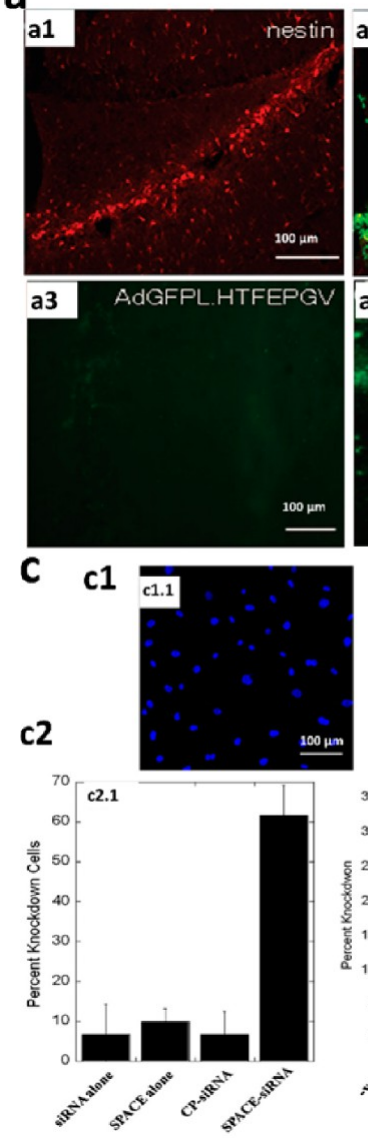
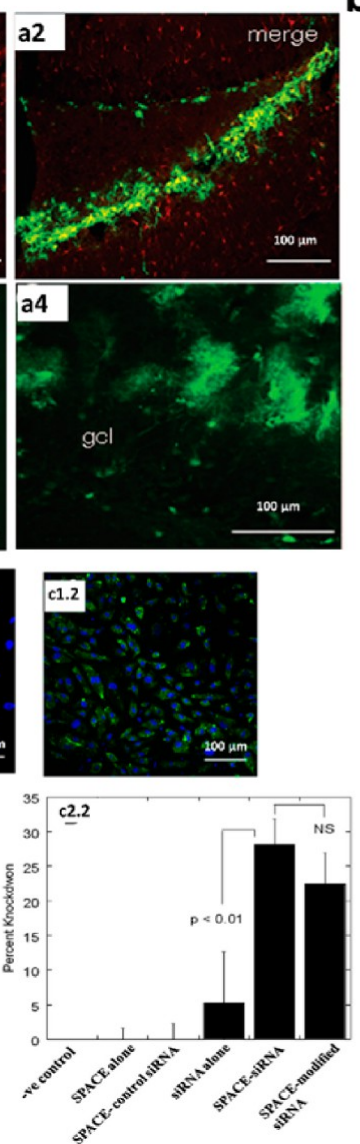

b b1

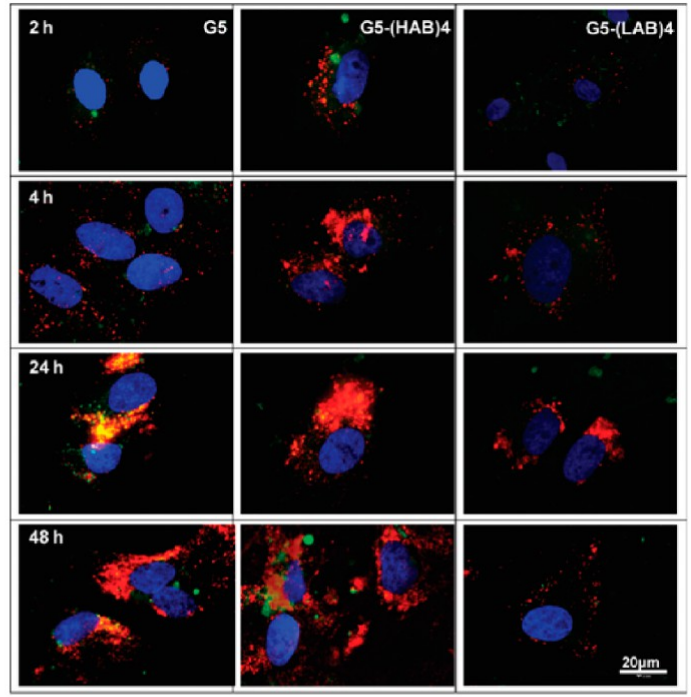

b2

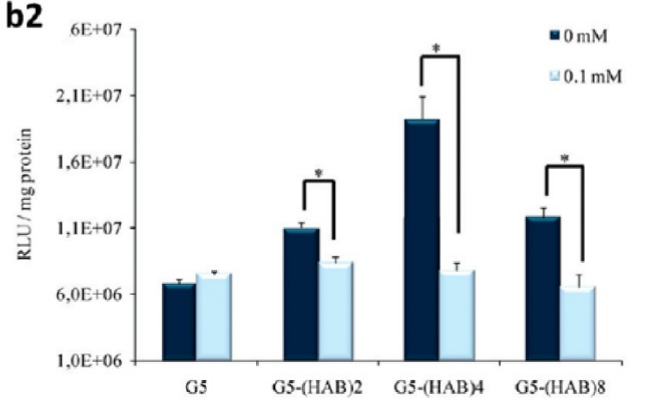

Figure 5. Cell-targeting gene delivery for selective and more efficient in vitro and in vivo gene therapy. (a) Selective transduction of NPCs in adult C57BL/6 mouse brain by capsid-mutated AdGFPL.VPTQSSG vector. Fluorescence-activated laser scanning microscopy images of brain sections analyzed by immunohistochemistry after injection of (a1, a2) PEGylated AdGFPL.VPTQSSG, (a3) AdGFPL.HTFEPGV, (a4) AdGFP into the dentate gyrus of adult mice. Nestin (red fluorescence, a1) and merge (yellow a2). Abbreviations: AdGFP, adenoviral vector that expresses GFP; AdGFPL, adenoviral vector that expresses GFP-Luc; VPTQSSG, NPC-specific binding peptide; HTFEPGV, unspecific peptide; gcl, granular cell layer. Reprinted and adapted with permission from ref 57. Copyright 2007 John Wiley and Sons. (b) Targeted gene delivery using PAMAM dendrimers functionalized with MSCs binding peptides. (b1) Fluorescence microscopy images showing intracellular localization of (RITC)-labeled pDNA (red) in MSCs transfected with native dendrimers (unconjugated, G5) and dendrimers conjugated with four peptide arms [G5-(HAB)4, G5-(LAB)4] as vectors. The endosomal-lysosomal system was stained with LysoSensor Green DND-189 (green), and the nucleus with DAPI (blue). (b2) Luc gene expression obtained with dendrimers (native and conjugated with 2, 4, and $8 \mathrm{HAB}$ peptide arms) with and without presaturation of cell receptors by $\mathrm{HAB}$ peptide $(0.1 \mathrm{mM})$. Reprinted with permission from ref 93. Copyright 2010 American Chemical Society. (c) Delivery of siRNA into the skin and cells using SPACE peptide. (c1) Confocal microscopy images of human umbilical vein endothelial cells (HUVECs; nuclei stained in blue) treated with (c1.1) PBS (control), (c1.2) FITC-labeled SPACE peptide for $24 \mathrm{~h}$. (c2) Delivery of siRNA in vitro and in vivo. (c2.1) Percentage of knockdown of GFP in GFP-expressing endothelial cells. (c2.2) Percentage of knockdown of interleukin-10 (IL-10) in mice after $24 \mathrm{~h}$ of treatment. Reprinted with permission from ref 60. Copyright 2011 United States National Academy of Sciences.

of a peptide specific for the principal component of the cartilage matrix (collagen II $\alpha 1$, Table 1). ${ }^{40}$ After intra-articular injection in a mouse model, conjugation of the peptide to nanoparticles led to higher retention of the nanoparticles within the ECM of articular cartilage ( 72 -fold increase after $48 \mathrm{~h}$ ) when compared to nanoparticles functionalized with a nontargeted (control) peptide. Such an approach could be used to deliver and localize specific GFs for cartilage repair.

Nanobiomaterial Carriers Functionalized with Peptide Ligands Identified by Phage Display for Genetic Manipulation of Cells in Vitro and in Vivo. The field of gene therapy for tissue repair and regeneration would benefit considerably from the isolation of peptides that can target endocytosing receptors on specific cells. ${ }^{90}$ Internalization of gene delivery systems is a prerequisite for efficient transgene expression. Peptides that can be internalized by cells are likely to be useful for nonviral-mediated gene delivery, where some current systems do not have the ability to enter the cells (e.g., polycation-DNA complexes). Gene therapy vectors could be modified with specific peptide ligands and delivered intravenously to transfect only specific cells, thus improving their efficacy and reducing the toxicity of the process. Therefore, many research groups have attempted to select cell-binding and cellpenetrating peptides ("homing peptides") using phage display for targeting specific cell types.

Targeted Gene Therapy. The use of phage display to identify cell-selective and cell-entry peptides as gene therapy vectors was first reported by Barry and colleagues in $1996 .^{92}$ They displayed 12- and 20-mer peptide libraries on pIII protein of fdTET phage and, by screening the libraries on mammalian cells, isolated cell-binding peptides. This was the first demonstration that cell-selective peptides could be identified by panning peptide phage libraries on cells, without prior information on the target receptors. However, in this pioneering 
work, they did not demonstrate the delivery of functional genes to the nucleus of specific mammalian cells.

In an attempt to develop in situ gene therapy, Schmidt et al. ${ }^{57}$ identified peptide ligands (Table 1) that selectively bound to mouse NPCs from phage display peptide libraries. They subsequently conjugated the identified peptides to wild-type capsid and capsid-mutated adenovirus (Ad) vectors and tested their targeting potential in vitro and in vivo, respectively. Peptides were shown to mediate Ad binding and infection of NPCs in vitro, and selective transduction of NPCs in the brain of adult C57BL/6 mice, as shown by the presence of nestin (Figure 5a1), an NPC-specific marker, colocalized with green fluorescent protein (GFP; Figure 5a2). Injection of a nonspecific peptide failed to transduce cells in the dentate gyrus, as seen by the absence of GFP expression (Figure 5a3), while injection of wildtype capsid AdGFP resulted in transduction of non-NPCs, such as astrocytes and neurons (Figure 5a4).

Using a nonviral vector (poly(amido)amine (PAMAM) dendrimers) functionalized with bone-targeting peptides, identified by phage display and having high (HAB) and low (LAB) affinity binding to mouse MSCs, Santos and co-workers ${ }^{93}$ were able to promote the uptake of plasmid DNA (pDNA) by rat MSCs (rMSCs). HAB peptide-functionalized dendrimers led to increased accumulation of pDNA inside cells (Figure 5b1). In addition, the presence of $\mathrm{HAB}$ bone-targeting peptides on the dendrimer surface led to higher transfection levels than those obtained with native dendrimers, measured by luciferase (Luc) gene expression (Figure 5b2). Similarly, Ma and colleagues ${ }^{53}$ used a rMSC-homing peptide, identified by phage display (Table 1), to improve the targeting capability of protamine/ DNA lipoplex (LPD) liposomes to rMSCs for the delivery of the Sleeping Beauty (SB) transposon plasmid. They also incorporated the positively charged nuclear localization signal (NLS) peptide to promote translocation of gene materials into the nucleus. By combining rMSC-targeting ability and NLS peptide into LPD liposomes, the transfection efficiency was improved, in comparison to control peptides (randomly selected), by enhancing the receptor-mediated endocytosis of LPD liposomes and their accumulation in the nucleus. They showed that the SB transposon and targeting LPD system did not result in cell toxicity and did not promote osteogenic differentiation, suggesting the potential of this system for effective nonviral gene delivery in stem cell therapy.

By panning on porcine skin, Hsu and Mitragotri ${ }^{60}$ identified a peptide by phage display, named the skin permeating and cell entering (SPACE) peptide, to enable the diffusion of macromolecules across the stratum corneum into the epidermis and dermis. The peptide also showed the ability to translocate the membrane of skin cells, such as keratinocytes, fibroblasts, and endothelial cells (Figure $5 \mathrm{cl}$ ). The efficacy of the peptide to carry and release small interfering RNA (siRNA) for GFP (Figure 5c2.1) and IL-10 was tested in vitro and in vivo (Figure 5c2.2), respectively. Peptide conjugation with siRNA led to enhanced cell penetration and absorption into the skin and knockdown of corresponding protein targets. On the contrary, however, no substantial knockdown was obtained with only siRNA or SPACE or using control siRNA conjugated with SPACE or a control peptide.

Using a cartilage affinity peptide (CAP, Table 1$)$ conjugated with polyethylenimine (PEI) for DNA complexation (CAP-PEI/DNA) in in vivo cartilage-targeted gene delivery, Pi et al. ${ }^{48}$ showed that the CAP-functionalized vector led to a 9-fold higher transfection efficiency in cartilage than the scrambled peptide-conjugated vector.
However, no statistical difference in the transfection efficiency was observed in the synovium between the vectors modified with targeted (CAP) and control (scrambled) peptide.

The identification of peptide ligands binding to receptors in organelles of mammalian cells (Table 1) was recently reported. ${ }^{61}$ When chemically fused to penetratin, the identified peptide was internalized and localized in the mitochondria, promoting cell death. This study demonstrated the further utility of phage display for subcellular targeted drug delivery. Advances in targeted intracellular delivery selectivity also has implications for $\mathrm{RM}$, namely, in RNA interference (RNAi)-based anabolic therapies, such as the delivery of osteogenic siRNAs specifically to osteoblasts (bone cells), aimed at promoting bone formation. ${ }^{94,95}$

Imaging Probes Functionalized with Peptide Ligands Identified by Phage Display for Targeted Imaging of Cells and Tissues in Vitro and in Vivo. Clinical imaging is an essential tool in RM. First, it is important to characterize the status of the damaged/diseased tissue for pretreatment planning, and second, it is necessary to evaluate the risks and efficacy of the therapy (post-transplant assessment). ${ }^{96}$

Injured and Regenerating Host Environment. The environment in injured or diseased tissues is not favorable for transplanted cells. Some tissues produce scars (e.g., fibrotic scar, glial scar) after injury, and this scarring process prevents engraftment of stem cells with the neighboring tissues. Additional factors that contribute for a hostile condition in damaged tissues include the presence of inflammatory cytokines and limited oxygen and nutrient delivery. Aging of the stem cell niche and corresponding alteration of the acellular components can cause a loss of stem cell functionality. Thus, imaging of the niche properties, including tissue architecture (structural integrity/morphological alterations) and molecular and cellular composition (e.g., ECM, GFs, cell populations), could be beneficial for ensuring that cells are hosted in a healthy environment (non-necrotic tissues). Peptides can be combined with fluorophores or radioisotopes for the development of new probes, ${ }^{97}$ as they provide numerous benefits over antibodies; they have higher stability and lower immunogenicity and diffuse faster (owing to their small size allowing better tissue penetration) and are easier to synthesize.

Peptide sequences binding to major components of the ECM of tissues, such as collagen and HA (Table 1), have been identified by phage display. These sequences have been further developed into molecular probes to detect these macromolecules in different tissues (e.g., pericardium, skin).

HA plays a central role in the wound healing process and influences stem cell behavior. ${ }^{98,99}$ The HA-binding peptide, described as Pep-1 in the literature, has been biotinylated for identification of HA in the skin. ${ }^{42}$

To improve the affinity of a phage-derived collagen-binding peptide for detecting collagen I in tissues, and to improve contrast/detection, the Meijer group proposed a new approach to display phage-derived peptides on dendritic architectures. ${ }^{18,38}$ In this system, a dendron mimics the pentavalent head of the phage (Figure 6a1). The system was then tested using a collagenspecific 7-mer peptide against collagen type I from rat tails. ${ }^{38}$ Pentavalent display of collagen-binding peptides on dendrimer wedges enhanced the affinity for collagen 100-fold when compared with the monovalent peptide, ${ }^{38}$ allowing selective staining of collagen in tissues containing collagen arranged in different levels of organization (fibrils, fibers, fiber bundles). Using $0.6 \mu \mathrm{M}$ fluorescein-labeled pentameric peptide on pig parietal pericardium, a highly defined fibrous network with fibers of $2 \mu \mathrm{m}$ in thickness was observed (Figure 6a2). This result demonstrates 
a

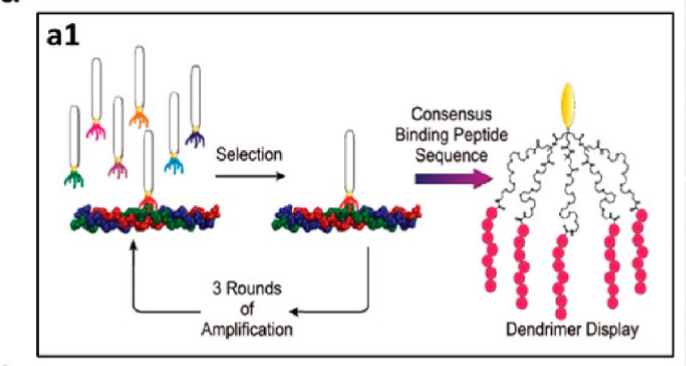

b
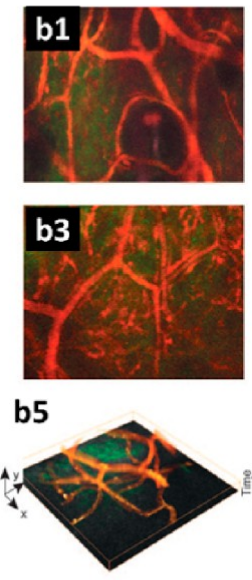

3D Reconstruction
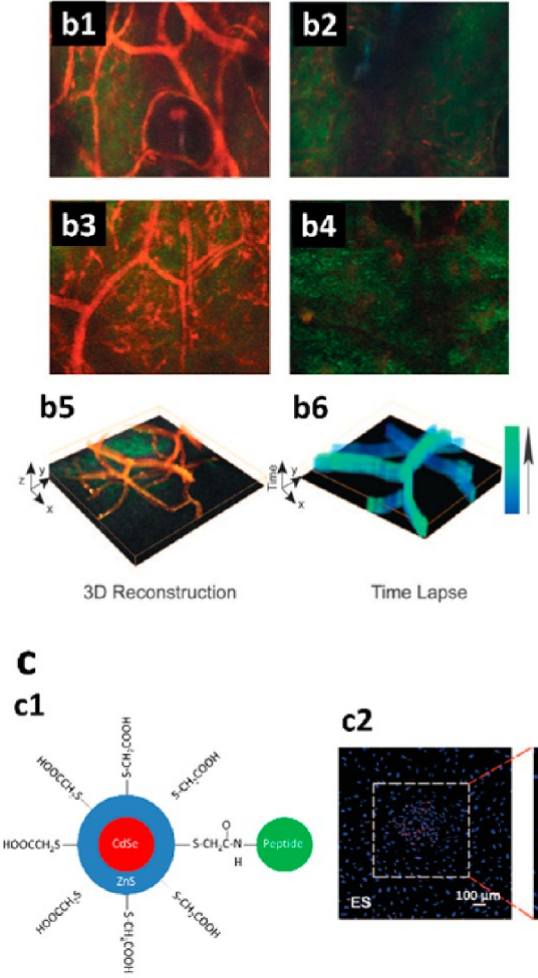
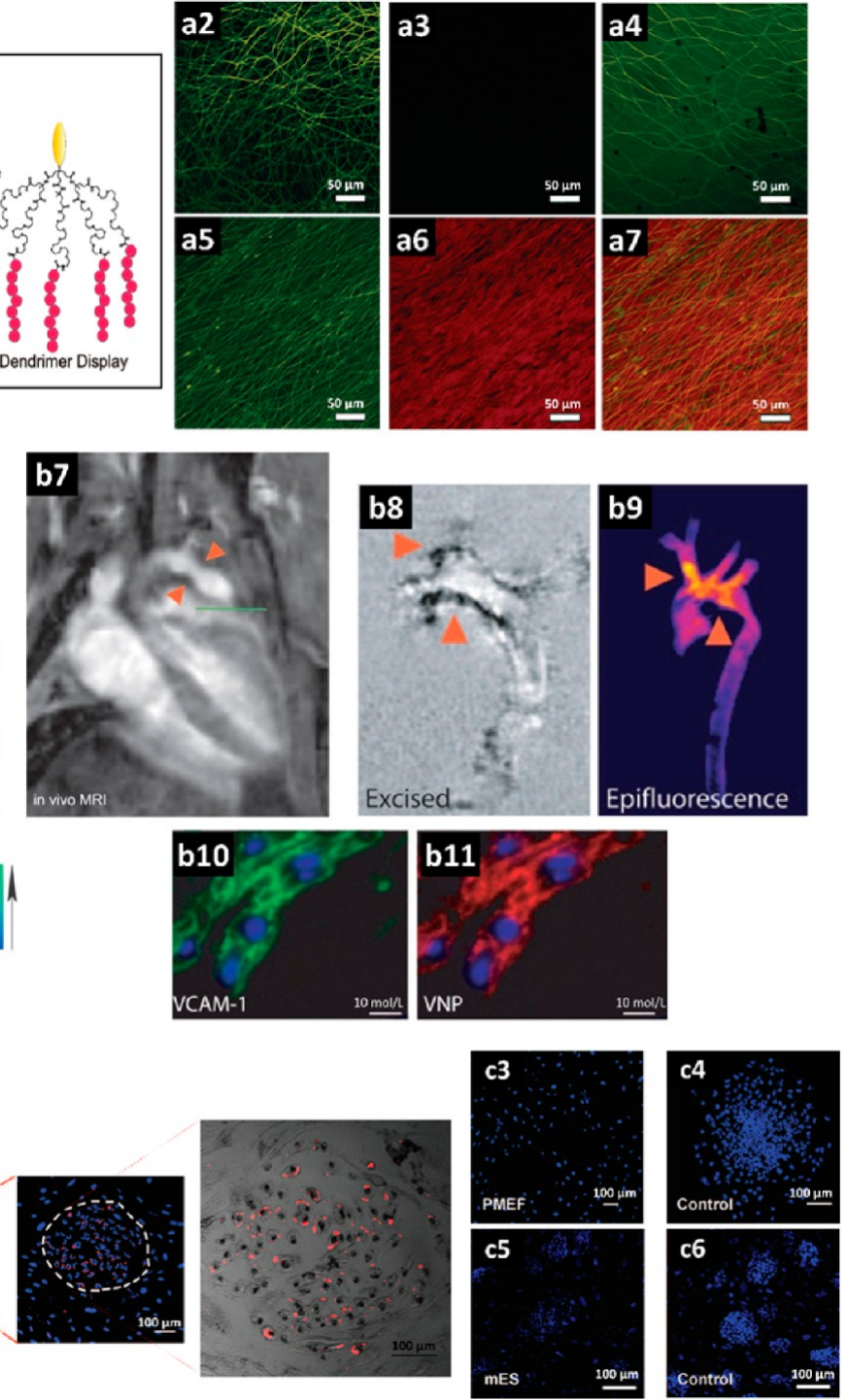

Figure 6. The application of phage-derived peptides for targeted imaging of cells and tissues in vitro and in vivo. (a) Collagen-I-specific probe based on collagen-binding peptide displayed on dendrimer edges. (a1) Collagen-binding peptide displayed on dendrimer edges resembling the typical pentavalent structure of phages. Laser scanning confocal microscopy images of pig parietal pericardium incubated with (a2) $0.6 \mu \mathrm{M}$ fluorescein-labeled peptide pentamer, (a3, a4) fluorescein-labeled monovalent collagen binding peptide at $0.6 \mu \mathrm{M}$ and $60 \mu \mathrm{M},(\mathrm{a} 5-\mathrm{a} 7) 6 \mu \mathrm{M}$ AlexaFluor568-labeled CNA35 (red) followed by $0.6 \mu \mathrm{M}$ fluorescein-labeled peptide pentamer (green) (costaining). Adapted with permission from ref 38 . Copyright 2009 American Chemical Society. (b) Detection of VCAM-1 by MRI and fluorescence imaging using VCAM-1-binding peptide (VP) and multimodal nanoparticles (VNPs) in atherosclerotic lesions. Intravital confocal microscopy images of mouse ear with (b1, b3) or without (b2, b4) mTNF- $\alpha$-induced inflammation at $4(\mathrm{~b} 1, \mathrm{~b} 2)$ and $24 \mathrm{~h}(\mathrm{~b} 3, \mathrm{~b} 4)$ after intravenous injection of VNP (red). Green color in the images is due to the tissue autofluorescence. Images obtained from the 3D stack reconstruction of the $\mathrm{Z}$ series (b5) from b3 and of the time series (b6) of VNP staining within the vessels (each time point is shown as an individual slice in the $\mathrm{Z}$ direction and the level of peptide staining (low to high) is given by the color scheme (blue to green)). In vivo (b7) and ex vivo (b8) MRI of the aorta of cholesterol-fed apoE ${ }^{-/-}$mice using gadolinium-protected graft copolymer (Gd-PGC) shows defined vascular lumen and aortic abnormalities such as narrowing (b7, arrows) and low signal changes caused by VNP localization (arrows, b8). (b9) Epifluorescence image of excised aorta using fluorescent labeled peptide confirming details observed in b8. (b10, b11) Immunofluorescence images of aorta sections revealing colocalization of VCAM-1 (green) and VNP (red). Nuclei in b10 and b11 are stained with DAPI (blue). Bars = $10 \mu \mathrm{m}$. Adapted with permission from ref 67. Copyright 2005 Lippincott Williams and Wilkins/Wolters Kluwer Health. (c) Targeted imaging of ESCs using peptideconjugated QDs. (c1) Schematic of CdSe-ZnS QDs conjugated with APWHLSSQYSRT peptide (green sphere). (c2) Fluorescence and bright-field microscopy images of ESCs (cell nuclei in blue, Hoechest 33258) after incubation with peptide-QDs (red) showing their binding to ESC colonies (inside dashed line). (c3) Fluorescence microscopy image of peptide QDs with PMEF cells which are used as a feeder layer (outside dashed line). $(c 4, c 6)$ Fluorescence microscopy images of cells with free QDs (without conjugated peptides, control). (c5) Fluorescence microscopy images mESCs peptide-conjugated QDs. Adapted with permission from ref 56. Copyright 2010 Public Library of Science.

that the identified peptide, derived from rat collagen, also binds to collagen of different species. The monovalent form of the collagen binding peptide at the same concentration (Figure 6a3) did not allow collagen detection. Comparable collagen fibrillar structures were visualized as for the pentameric peptide when using $60 \mu \mathrm{M}$ of the monovalent peptide, but the intensity of background fluorescence was higher (Figure 6a4). A naturally occurring collagen binding protein (CNA35) was used as a comparison with the fluorescein-labeled pentavalent peptide. Both probes allowed visualization of collagen fibers with the 
same orientation, but the pentameric peptide led to a more specific stain when compared to CNA35 (Figure 6a5-a7).

Optical and nuclear imaging agents could be coupled to ECM-binding peptides to allow clinical imaging and characterize organ/tissue morphology (e.g., degraded matrix) before and after the therapies (matrix formation as a result of regeneration).

Lee and collaborators reported the results of panning against stabilin- $2^{64}$ (Table 1), known to be produced by activated macrophages and also strongly expressed in smooth muscle cells and endothelial cells of atherosclerotic lesions. The identified peptide sequence was found to bind specifically to stabilin- 2 and localize to atherosclerotic plaques in vivo. In addition, the peptide was conjugated with fluorescently tagged glycol chitosan nanoparticles and administered to apolipoprotein E-deficient mice through direct injection into the left ventricles. The peptide-conjugated nanoparticles were found to accumulate in the atherosclerotic lesions, while control nanoparticles were not visible. These studies revealed peptide ligands that can be used for selective molecular imaging of atherosclerosis.

Phage display-derived vascular adhesion molecule 1 (VCAM-1)specific peptide has allowed targeted imaging of activated endothelium under inflammatory conditions by fluorescence imaging and magnetic resonance imaging (MRI, Figure $6 \mathrm{~b}$ ) and has enabled the visualization of structural abnormalities in the aortic wall. ${ }^{67}$ Similar probes could be developed against pro-inflammatory cytokines implicated in tissue injury (e.g., tumor necrosis factor $\alpha$ (TNF- $\alpha$ ); interleukin 1- $\beta$ (IL-1 $\beta)$ ) to characterize tissue functionality and delineate the feasibility of the cell therapy.

Phage-derived peptides would be also useful to measure regeneration outcomes, such as angiogenesis and formation of new tissue. For example, angiogenic vasculature has been assessed by imaging the binding of RGD-containing peptides conjugated to PET imaging agents to specific integrins on the cell surface. ${ }^{100}$ Using the above-described probes, the production and structure of tissue-specific ECM components (e.g., collagen II in cartilage or HAP in bone or tooth) could be visualized independently and noninvasively through different imaging modes. Similarly, the functionality of newly formed tissue could be detected by assessing the presence of specific cell populations using ligands for phenotype-specific markers. The discovery of novel peptide ligands for the development of molecular imaging probes with enhanced specificity for detecting constant changes in the environment of damaged and regenerating tissues would greatly benefit cell-based therapies.

Cell Labeling/Tracking. Tracking the distribution, function, and fate of transplanted cells using noninvasive imaging is an important goal in RM. The ability to assess cell localization, viability, growth, differentiation, and engraftment in the host would be extremely valuable. However, cells administered into humans cannot be tracked unless they are first labeled in vitro. Cells can be labeled directly, using chemical agents, or indirectly, by inclusion of reporter genes. Cell labeling methods should be specific, nontoxic, and stable (i.e., label agents should remain in the target cells for suitable periods of time to allow correlation with cell numbers).

Several cell-binding peptides have been identified, with the isolation of stem-cell binding peptides being of particular utility for RM applications ${ }^{100}$ (Table 1). These peptides can be exploited for cell labeling/imaging and isolation purposes. For example, Ma's group ${ }^{54-56,59}$ used phage-derived cell-binding peptides conjugated with quantum dots (QDs, light emitting agents) to specifically label ESCs. By panning on rhesus macaque ESCs, they identified the sequence, APWHLSSQYSRT
(Table 1), with high affinity for undifferentiated ESCs. They subsequently conjugated the peptide to $\mathrm{CdSe}-\mathrm{ZnS}$ QDs (Figure $6 \mathrm{cl}$ ) and showed that the conjugates could bind efficiently and selectively to ESCs, with no binding observed for other cell types (primary mouse embryonic fibroblast, PMEF, Figure 6c3) and ESCs from other species (mouse ESCs, Figure 6c5), or when control QDs were used (Figure 6c4,c6). The E7 peptide (Table 1), known to bind hMSCs, was conjugated to gadolinium(Gd)-1,4,7,10-tetraazacyclododecane1,4,7,10-tetraacetic acid (DOTA) for labeling MSCs for MRI. ${ }^{101}$ In vitro studies showed Gd-DOTA-E7 yielded minimum labeling efficacy but good contrast enhancement.

Identification of peptides that interact with stage-specific cellular targets (e.g., differentiation stage) would be useful for assessing molecular profiles of cells in vivo (e.g., imaging differentiation of transplanted cells). Zhao et al. ${ }^{59}$ identified a peptide with the ability to bind NSCs derived from rhesus monkey ESCs and used this peptide combined with QDs for cell imaging. This study described for the first time the use of phage-derived peptides for studying ESC differentiation by optical imaging.

A problem with these imaging agents is that the signal cannot be used to distinguish live from dead cells, and it is not certain whether or not these probes will be retained by cells in the long term. Previous studies indicated that after cell death, contrast agents can be passed to host cells, producing false positives. ${ }^{102,103}$ Thus, monitoring stem cell fate may require a combination of complementary imaging methods, such as fluorescence and magnetic resonance, to allow the correlation between anatomical localization of labeled stem cells and cell viability. Reporter genes represent a potential solution to this issue, although this can pose additional safety and regulatory problems. Phage display could also contribute toward the development of molecular probes that would bind only viable cells resulting in the release of the label after cell death. Since dead cells have a compromised cell membrane, it might be possible to identify a specific ligand for live cells by panning on dead cells first (preclearing).

\section{CONCLUDING REMARKS AND OUTLOOK}

The ability to display a large and highly diverse collection of random peptide sequences on the coat proteins of phages offers the possibility to discover new interactions with useful targets, often without knowing their structure. The field of phage display technology has evolved significantly from when it was first developed for mapping interactions between proteins and has expanded into other areas that benefit from the discovery of new interacting ligands. This review demonstrates the usefulness of phage display in the emerging field of RM, suggesting opportunities for further applications. However, we must consider the challenges associated with phage-derived peptides before their translation into useful RM tools. When used as linear monomers, isolated peptide ligands usually show poor affinity for their targets, as this presentation differs significantly from the original display (valency and orientation) on the phage particles. Multimerization (conjugation of multiple peptide copies to a surface of a nanoparticle, like dendrimers, liposomes, or micelles) has been proven to increase affinity of the ligand for its targets, but the affinity of these multivalent peptide platforms also depends on the density of receptors on the cell periphery. In addition, when displayed on the phage, peptides are fused to the coat protein through the $\mathrm{C}$-terminus and have a free $\mathrm{N}$-terminus. This feature is not always taken into account when presenting phage-derived peptides into multivalent carriers. This stresses the importance of the use of biomaterials engineering to enable the peptides to be 
displayed in the precise and optimal multimeric conformation for interaction with cell receptors. Presenting peptides within a biomaterials platform also provides protection against proteolytic degradation, which is essential for prolonging their half-life in vivo.

It should be noted that many of the sequences described in this review were obtained from targets derived from multiple species. Thus, it is necessary to confirm if these peptides also bind to the same targets in humans. Although cell-binding peptides can be used without knowing their specific receptors, identification of their cellular targets is essential for gaining clinical approval, as emphasized by Gray and Brown. ${ }^{100}$ It is also useful for obtaining information about the heterogeneity of the protein repertoire on the cell surface and how it varies among cell types and states.

To accelerate the safe application of phage-derived peptides within the field of RM, the next logical steps will be their further optimization and validation in vitro and in vivo, in terms of affinity, specificity, activity, stability, and biodistribution.

While binding has been the basis for peptide screening in phage display, selection based on function might be useful for certain RM approaches (e.g., controlled stem cell differentiation). In a recent perspective article, ${ }^{104}$ Lerner highlighted the potential of intracellular combinatorial (unbiased) antibody libraries as a discovery tool to select antibodies able to bind unknown receptors or to identify new roles of common receptors. They showed that infection of BMSCs with unbiased libraries led to the selection of various antibody agonists that induced proliferation of cells, or proliferation followed by differentiation or trans-differentiation into neural cells, indicating different roles for identified receptors in differentiation. These studies suggest new ways to regulate cell fates by selecting cellbinding antibodies or peptides on the basis of function (e.g., ability to activate a given receptor involved in inducing differentiation).

With further advances in biology and RM, there are enormous opportunities for phage display to contribute tools for research and applications in such fields. Led by a vibrant research community in biology and RM, phage display technology will have a broad range of applications in the future.

\section{ASSOCIATED CONTENT}

\section{S Supporting Information}

The Supporting Information is available free of charge on the ACS Publications website at DOI: 10.1021/acschembio.5b00717.

Table S1 shows the follow-up analysis on the subsequent applications of peptide sequences listed in Table 1 (PDF)

\section{AUTHOR INFORMATION}

\section{Corresponding Author}

*Tel.: +44 (0)20 7882 5502. Fax: +44 (0)20 7882 3390. E-mail: h.azevedo@qmul.ac.uk.

\section{Notes}

The authors declare no competing financial interest.

\section{ACKNOWLEDGMENTS}

This work was supported by national funds through the Portuguese Foundation for Science and Technology under the scope of the project PTDC/EBB-BIO/114523/2009 and by the European Regional Development Fund (ERDF) through the Operational Competitiveness Programme "COMPETE" (FCOMP-01-0124-FEDER-014758). The authors are also thankful for the financial support of the Portuguese Foundation for Science and Technology under the strategic funding of UID/ $\mathrm{BIO} / 04469 / 2013$ unit and RECI/BBB-EBI/0179/2012
(FCOMP-01-0124-FEDER-027462) and the European Union under the Marie Curie Career Integration Grant SuprHApolymers (PCIG14-GA-2013-631871). We thank L. Kluskens, from the Center of Biological Engineering at the University of Minho (Portugal) for his expert opinion on phage display and valuable comments during the reading of the manuscript. We are also very grateful to A. Mata from the School of Engineering \& Materials Science at Queen Mary University of London for his insightful comments and suggestions on the manuscript.

\section{KEYWORDS}

Bacteriophages (or phages): viruses that specifically infect bacterial cells and consist of an outer protein capsid enclosing genetic material (dsDNA - vast majority; ssDNA; ssRNA; or dsRNA - very rare; with either circular or linear arrangement) Biomaterials: synthetic or natural materials designed to interact with tissues and organs in the body to monitor and/or restore their functions

Nanotechnology: technology that involves imaging, measuring, modeling, and manipulating materials on the nanometer scale (i.e., $10^{-9} \mathrm{~m}$ ), typically in the range of 1 to $100 \mathrm{~nm}$

Peptide ligand: a peptide sequence which binds with high affinity and specificity to a particular target

Phage display panning: combinatorial process of displaying random peptide sequences or engineered proteins, fused to the coat proteins on the surface of phages, to identify peptides or proteins (out of billions of candidates) that bind specifically and selectively to a defined or unknown target

Phage display peptide library: collection of phage particles displaying random peptides containing as many as $10^{9}$ combinations

Regenerative medicine: new field in the health sciences dedicated to the regeneration of tissues or organs by applying specific cell populations or biomaterial scaffolds, alone or combined, to stimulate the intrinsic healing ability of the body and promote endogenous repair

Targeted delivery: method of delivering a therapeutic (drug, bioactive protein, or gene) or imaging (probe) agent to a specific site in the body, which optimizes its therapeutic or imaging index by restricting its pharmacological or imaging activity to the target tissue or organ, and reducing potential exposure in unwanted sites

Ad: adenovirus

ALP: alkaline phosphatase

ASCs: adipose stromal cells

ATs: alkanethiols

BC-1: bifunctional peptide 1

bFGF: basic fibroblast growth factor

BMHP: bone marrow homing peptide

BMP-2: bone morphogenetic protein 2

BMSCs: bone marrow stem cells

BRASIL: biopanning and rapid analysis of selective interactive ligands

C3: complement 3

CAP: chondrocyte-affinity peptide

cBMHP: cyclic BMHP

cDNA: complementary DNA

Col: collagen

DMEM: Dulbecco's modified eagle medium

DNA: DNA

DOTA: 1,4,7,10-tetraazacyclododecane-1,4,7,10-tetraacetic acid

ds: double stranded 
EC $_{50}$ : half maximal effective concentration

ECM: extracellular matrix

ELISA: enzyme-linked immunosorbent assay

ESCs: embryonic stem cells

FDA: U.S. Food and Drug Administration

FITC: fluorescein isothiocyanate

GAG: glycosaminoglycan

Gd-PGC: gadolinium-protected graft copolymer

GF: growth factor

GFP: green fluorescent protein

HA: hyaluronic acid (or hyaluronan)

HAB: high affinity binding

hACs: human articular chondrocytes

HAP: hydroxyapatite

H\&E: hematoxylin and eosin

HUVECs: human umbilical vein endothelial cells

IC $_{50}$ : half inhibitory concentration

IL-1: interleukin 1

IL-10: interleukin 10

$\mathrm{K}_{\mathbf{D}}$ : dissociation constant

LAB: low affinity binding

MRI: magnetic resonance imaging

mRNA: mRNA

MSCs: mesenchymal stem cells

mTNF- $\alpha$ : murine TNF- $\alpha$

NGS: next generation sequencing

NLS: nuclear localization signal

NPCs: neural progenitor cells

NSCs: neural stem cells

OA: osteoarthritis

ODM: osteogenic differentiation medium

PA: peptide amphiphile

PAMAM: poly(amido)amine

PCL: polycaprolactone

pDNA: plasmid DNA

PEG: polyethylene glycol

PEI: polyethylenimine

PET: positron emission tomography

PMEF: primary mouse embryonic fibroblast

QDs: quantum-dots

RGD: arginine-glycine-aspartic acid

RHAMM: receptor for hyaluronan-mediated motility

rhBMP-2: recombinant form of human BMP-2

$\mathbf{R M}$ : regenerative medicine

rMSC: rat MSCs

RNA: ribonucleic acid

RNAi: RNA interference

S2P: stabilin-2 peptide

SA: streptavidin

SAM: self-assembled monolayer

SEM: scanning electron microscopy

siRNA: small interfering RNA

SPACE: skin permeating and cell entering

ss: single stranded

TGF- $\boldsymbol{\beta}$ : transforming growth factor $\beta$

TGF- $\beta$ R: transforming growth factor $\beta$ receptor

TNF: tumor necrosis factor

VCAM-1: vascular cell adhesion molecule 1

VNP: VHSPNKK-modified magnetofluorescent nanoparticle

\section{REFERENCES}

(1) Mason, C., and Dunnill, P. (2008) A brief definition of regenerative medicine. Regener. Med. 3, 1-5.
(2) Smith, G. P. (1985) Filamentous fusion phage: novel expression vectors that display cloned antigens on the virion surface. Science 228, 1315-1317.

(3) Deutscher, S. L. (2010) Phage display in molecular imaging and diagnosis of cancer. Chem. Rev. 110, 3196-3211.

(4) Hamzeh-Mivehroud, M., Alizadeh, A. A., Morris, M. B., Church, W. B., and Dastmalchi, S. (2013) Phage display as a technology delivering on the promise of peptide drug discovery. Drug Discovery Today 18, 1144-1157.

(5) Krumpe, L. R., and Mori, T. (2006) The Use of Phage-Displayed Peptide Libraries to Develop Tumor-Targeting Drugs. Int. J. Pept. Res. Ther. 12, 79-91.

(6) Smith, G. P., and Petrenko, V. A. (1997) Phage Display. Chem. Rev. 97, 391-410.

(7) Petrenko, V. (2008) Evolution of phage display: from bioactive peptides to bioselective nanomaterials. Expert Opin. Drug Delivery 5, $825-836$.

(8) Shiba, K. (2010) Exploitation of peptide motif sequences and their use in nanobiotechnology. Curr. Opin. Biotechnol. 21, 412-425.

(9) Wilson, D. R., and Finlay, B. B. (1998) Phage display: applications, innovations, and issues in phage and host biology. Can. J. Microbiol. 44, 313-329.

(10) Bratkovic, T. (2010) Progress in phage display: evolution of the technique and its application. Cell. Mol. Life Sci. 67, 749-767.

(11) Pande, J., Szewczyk, M. M., and Grover, A. K. (2010) Phage display: concept, innovations, applications and future. Biotechnol. Adv. $28,849-858$

(12) Paschke, M. (2006) Phage display systems and their applications. Appl. Microbiol. Biotechnol. 70, 2-11.

(13) Sergeeva, A., Kolonin, M. G., Molldrem, J. J., Pasqualini, R., and Arap, W. (2006) Display technologies: application for the discovery of drug and gene delivery agents. Adv. Drug Delivery Rev. 58, 1622-1654.

(14) Smothers, J. F., Henikoff, S., and Carter, P. (2002) Phage display: Affinity selection from biological libraries. Science 298, 621-622.

(15) Barbas, C. F., Burton, D. R., Scott, J. K., and Silverman, G. J. (2001) Phage Display: A Laboratory Manual, Cold Spring Harbor Laboratory Press, New York.

(16) Clarkson, T., and Lowman, H. B. (2004) Phage Display: A Practical Approach, Oxford University Press, Oxford.

(17) Hamilton, P. T., Jansen, M. S., Ganesan, S., Benson, R. E., HydeDeruyscher, R, Beyer, W. F., Gile, J. C., Nair, S. A., Hodges, J. A., and Gron, H. (2013) Improved bone morphogenetic protein-2 retention in an injectable collagen matrix using bifunctional peptides. PLoS One 8, e70715.

(18) Bastings, M. M., Helms, B. A., van Baal, I., Hackeng, T. M., Merkx, M., and Meijer, E. W. (2011) From phage display to dendrimer display: insights into multivalent binding. J. Am. Chem. Soc. 133, 6636-6641.

(19) Hajduczki, A., Majumdar, S., Fricke, M., Brown, I. A., and Weiss, G. A. (2011) Solubilization of a membrane protein by combinatorial supercharging. ACS Chem. Biol. 6, 301-307.

(20) Giordano, R. J., Cardo-Vila, M., Lahdenranta, J., Pasqualini, R., and Arap, W. (2001) Biopanning and rapid analysis of selective interactive ligands. Nat. Med. 7, 1249-1253.

(21) Derda, R., Musah, S., Orner, B. P., Klim, J. R., Li, L., and Kiessling, L. L. (2010) High-throughput discovery of synthetic surfaces that support proliferation of pluripotent cells. J. Am. Chem. Soc. 132, 12891295.

(22) Arap, W., Kolonin, M. G., Trepel, M., Lahdenranta, J., Cardo-Vila, M., Giordano, R. J., Mintz, P. J., Ardelt, P. U., Yao, V. J., Vidal, C. I., Chen, L., Flamm, A., Valtanen, H., Weavind, L. M., Hicks, M. E., Pollock, R. E., Botz, G. H., Bucana, C. D., Koivunen, E., Cahill, D., Troncoso, P., Baggerly, K. A., Pentz, R. D., Do, K. A., Logothetis, C. J., and Pasqualini, R. (2002) Steps toward mapping the human vasculature by phage display. Nat. Med. 8, 121-127.

(23) Chen, Y., Shen, Y., Guo, X., Zhang, C., Yang, W., Ma, M., Liu, S., Zhang, M., and Wen, L. P. (2006) Transdermal protein delivery by a coadministered peptide identified via phage display. Nat. Biotechnol. 24, $455-460$. 
(24) Pasqualini, R., and Ruoslahti, E. (1996) Organ targeting in vivo using phage display peptide libraries. Nature 380, 364-366.

(25) Trepel, M., Arap, W., and Pasqualini, R. (2002) In vivo phage display and vascular heterogeneity: implications for targeted medicine. Curr. Opin. Chem. Biol. 6, 399-404.

(26) Nowakowski, G. S., Dooner, M. S., Valinski, H. M., Mihaliak, A. M., Quesenberry, P. J., and Becker, P. S. (2004) A specific heptapeptide from a phage display peptide library homes to bone marrow and binds to primitive hematopoietic stem cells. Stem Cells 22, 1030-1038.

(27) Matochko, W. L., Chu, K., Jin, B., Lee, S. W., Whitesides, G. M., and Derda, R. (2012) Deep sequencing analysis of phage libraries using Illumina platform. Methods 58, 47-55.

(28) t Hoen, P. A. C., Jirka, S. M., Ten Broeke, B. R., Schultes, E. A., Aguilera, B., Pang, K. H., Heemskerk, H., Aartsma-Rus, A., van Ommen, G. J., and den Dunnen, J. T. (2012) Phage display screening without repetitious selection rounds. Anal. Biochem. 421, 622-631.

(29) Ernst, A., Gfeller, D., Kan, Z., Seshagiri, S., Kim, P. M., Bader, G. D., and Sidhu, S. S. (2010) Coevolution of PDZ domain-ligand interactions analyzed by high-throughput phage display and deep sequencing. Mol. BioSyst. 6, 1782-1790.

(30) Rentero Rebollo, I., Sabisz, M., Baeriswyl, V., and Heinis, C. (2014) Identification of target-binding peptide motifs by highthroughput sequencing of phage-selected peptides. Nucleic Acids Res. 42, e169.

(31) Robins, W. P., Faruque, S. M., and Mekalanos, J. J. (2013) Coupling mutagenesis and parallel deep sequencing to probe essential residues in a genome or gene. Proc. Natl. Acad. Sci. U. S. A. 110, E848857.

(32) Zhang, H., Torkamani, A., Jones, T. M., Ruiz, D. I., Pons, J., and Lerner, R. A. (2011) Phenotype-information-phenotype cycle for deconvolution of combinatorial antibody libraries selected against complex systems. Proc. Natl. Acad. Sci. U. S. A. 108, 13456-13461.

(33) Huang, J., Ru, B., and Dai, P. (2011) Bioinformatics resources and tools for phage display. Molecules 16, 694-709.

(34) Huang, J., Ru, B., Zhu, P., Nie, F., Yang, J., Wang, X., Dai, P., Lin, H., Guo, F. B., and Rao, N. (2012) MimoDB 2.0: a mimotope database and beyond. Nucleic Acids Res. 40, 271-277.

(35) Sahu, A., Kay, B. K., and Lambris, J. D. (1996) Inhibition of human complement by a C3-binding peptide isolated from a phagedisplayed random peptide library. J. Immunol 157, 884-891.

(36) Ricklin, D., and Lambris, J. D. (2008) Compstatin: a complement inhibitor on its way to clinical application. Adv. Exp. Med. Biol. 632, 262281.

(37) Nilsson, B., Ekdahl, K. N., Mollnes, T. E., and Lambris, J. D. (2007) The role of complement in biomaterial-induced inflammation. Mol. Immunol. 44, 82-94.

(38) Helms, B. A., Reulen, S. W., Nijhuis, S., de Graaf-Heuvelmans, P. T., Merkx, M., and Meijer, E. W. (2009) High-affinity peptide-based collagen targeting using synthetic phage mimics: from phage display to dendrimer display. J. Am. Chem. Soc. 131, 11683-11685.

(39) Gron, H., and Duffin, D. Methods and compositions for promoting localization of pharmaceutically active agents to bone. Patent 2008/0268015A1, 2008.

(40) Rothenfluh, D. A., Bermudez, H., O’Neil, C. P., and Hubbell, J. A. (2008) Biofunctional polymer nanoparticles for intra-articular targeting and retention in cartilage. Nat. Mater. 7, 248-254.

(41) Chan, J. M., Zhang, L., Tong, R., Ghosh, D., Gao, W., Liao, G., Yuet, K. P., Gray, D., Rhee, J. W., Cheng, J., Golomb, G., Libby, P., Langer, R., and Farokhzad, O. C. (2010) Spatiotemporal controlled delivery of nanoparticles to injured vasculature. Proc. Natl. Acad. Sci. U.S. A. 107, 2213-2218.

(42) Mummert, M. E., Mohamadzadeh, M., Mummert, D. I., Mizumoto, N., and Takashima, A. (2000) Development of a peptide inhibitor of hyaluronan-mediated leukocyte trafficking. J. Exp. Med. 192, 769-779.

(43) Roy, M. D., Stanley, S. K., Amis, E. J., and Becker, M. L. (2008) Identification of a highly specific hydroxyapatite-binding peptide using phage display. Adv. Mater. 20, 1830-1836.
(44) Gungormus, M., Fong, H., Kim, I. W., Evans, J. S., Tamerler, C., and Sarikaya, M. (2008) Regulation of in vitro calcium phosphate mineralization by combinatorially selected hydroxyapatite-binding peptides. Biomacromolecules 9, 966-973.

(45) Chung, W. J., Kwon, K. Y., Song, J., and Lee, S. W. (2011) Evolutionary screening of collagen-like peptides that nucleate hydroxyapatite crystals. Langmuir 27, 7620-7628.

(46) Li, Z., Fan, J., Zhao, W., Jin, L., and Ma, L. (2011) The specific binding of peptide ligands to cardiomyocytes derived from mouse embryonic stem cells. J. Pept. Sci. 17, 771-782.

(47) Cheung, C. S., Lui, J. C., and Baron, J. (2013) Identification of chondrocyte-binding peptides by phage display. J. Orthop. Res. 31, $1053-1058$.

(48) Pi, Y., Zhang, X., Shi, J., Zhu, J., Chen, W., Zhang, C., Gao, W., Zhou, C., and Ao, Y. (2011) Targeted delivery of non-viral vectors to cartilage in vivo using a chondrocyte-homing peptide identified by phage display. Biomaterials 32, 6324-6332.

(49) Nie, J., Chang, B., Traktuev, D. O., Sun, J., March, K., Chan, L., Sage, E. H., Pasqualini, R., Arap, W., and Kolonin, M. G. (2008) IFATS collection: Combinatorial peptides identify alphaSbetal integrin as a receptor for the matricellular protein SPARC on adipose stromal cells. Stem Cells 26, 2735-2745.

(50) Daquinag, A. C., Zhang, Y., Amaya-Manzanares, F., Simmons, P. J., and Kolonin, M. G. (2011) An isoform of decorin is a resistin receptor on the surface of adipose progenitor cells. Cell Stem Cell 9, 74-86.

(51) Shao, Z., Zhang, X., Pi, Y., Wang, X., Jia, Z., Zhu, J., Dai, L., Chen, W., Yin, L., Chen, H., Zhou, C., and Ao, Y. (2012) Polycaprolactone electrospun mesh conjugated with an MSC affinity peptide for MSC homing in vivo. Biomaterials 33, 3375-3387.

(52) Balian, G. Bone Targeting Peptides. Patent 7,323,542, 2008

(53) Ma, K., Wang, D. D., Lin, Y., Wang, J., Petrenko, V., and Mao, C. (2013) Synergetic Targeted Delivery of Sleeping-Beauty Transposon System to Mesenchymal Stem Cells Using LPD Nanoparticles Modified with a Phage-Displayed Targeting Peptide. Adv. Funct. Mater. 23, 11721181.

(54) Zhao, W., Jin, L., Yuan, H., Tan, Z., Zhou, C., Li, L. S., and Ma, L. (2013) Targeting human embryonic stem cells with quantum dotconjugated phages. Sci. Rep. 3, 3134.

(55) Zhao, S., Zhao, W., and Ma, L. (2010) Novel peptide ligands that bind specifically to mouse embryonic stem cells. Peptides 31, 20272034.

(56) Lu, S., Xu, X., Zhao, W., Wu, W., Yuan, H., Shen, H., Zhou, C., Li, L. S., and Ma, L. (2010) Targeting of embryonic stem cells by peptideconjugated quantum dots. PLoS One 5, e12075.

(57) Schmidt, A., Haas, S. J., Hildebrandt, S., Scheibe, J., Eckhoff, B., Racek, T., Kempermann, G., Wree, A., and Putzer, B. M. (2007) Selective targeting of adenoviral vectors to neural precursor cells in the hippocampus of adult mice: new prospects for in situ gene therapy. Stem Cells 25, 2910-2918.

(58) Caprini, A., Silva, D., Zanoni, I., Cunha, C., Volonte, C., Vescovi, A., and Gelain, F. (2013) A novel bioactive peptide: assessing its activity over murine neural stem cells and its potential for neural tissue engineering. New Biotechnol. 30, 552-562.

(59) Zhao, W., Yuan, H., Xu, X., and Ma, L. (2010) Isolation and initial application of a novel peptide that specifically recognizes the neural stem cells derived from rhesus monkey embryonic stem cells. J. Biomol. Screening 15, 687-694.

(60) Hsu, T., and Mitragotri, S. (2011) Delivery of siRNA and other macromolecules into skin and cells using a peptide enhancer. Proc. Natl. Acad. Sci. U. S. A. 108, 15816-15821.

(61) Rangel, R., Guzman-Rojas, L., le Roux, L. G., Staquicini, F. I., Hosoya, H., Barbu, E. M., Ozawa, M. G., Nie, J., Dunner, K., Jr., Langley, R. R., Sage, E. H., Koivunen, E., Gelovani, J. G., Lobb, R. R., Sidman, R. L., Pasqualini, R, and Arap, W. (2012) Combinatorial targeting and discovery of ligand-receptors in organelles of mammalian cells. Nat. Commun. 3, 788.

(62) Behanna, H. A., Donners, J. J., Gordon, A. C., and Stupp, S. I. (2005) Coassembly of amphiphiles with opposite peptide polarities into nanofibers. J. Am. Chem. Soc. 127, 1193-1200. 
(63) Yayon, A., Aviezer, D., Safran, M., Gross, J. L., Heldman, Y., Cabilly, S., Givol, D., and Katchalski-Katzir, E. (1993) Isolation of peptides that inhibit binding of basic fibroblast growth factor to its receptor from a random phage-epitope library. Proc. Natl. Acad. Sci. U.S. A. 90, 10643-10647.

(64) Lee, G. Y., Kim, J. H., Oh, G. T., Lee, B. H., Kwon, I. C., and Kim, I. S. (2011) Molecular targeting of atherosclerotic plaques by a stabilin-2specific peptide ligand. J. Controlled Release 155, 211-217.

(65) Li, L., Orner, B. P., Huang, T., Hinck, A. P., and Kiessling, L. L. (2010) Peptide ligands that use a novel binding site to target both TGFbeta receptors. Mol. BioSyst. 6, 2392-2402.

(66) Michon, I. N., Penning, L. C., Molenaar, T. J., van Berkel, T. J., Biessen, E. A., and Kuiper, J. (2002) The effect of TGF-beta receptor binding peptides on smooth muscle cells. Biochem. Biophys. Res. Commun. 293, 1279-1286.

(67) Kelly, K. A., Allport, J. R., Tsourkas, A., Shinde-Patil, V. R., Josephson, L., and Weissleder, R. (2005) Detection of vascular adhesion molecule-1 expression using a novel multimodal nanoparticle. Circ. Res. 96, 327-336.

(68) Nahrendorf, M., Jaffer, F. A., Kelly, K. A., Sosnovik, D. E., Aikawa, E., Libby, P., and Weissleder, R. (2006) Noninvasive vascular cell adhesion molecule-1 imaging identifies inflammatory activation of cells in atherosclerosis. Circulation 114, 1504-1511.

(69) Yang, B., Yang, B. L., Savani, R. C., and Turley, E. A. (1994) Identification of a common hyaluronan binding motif in the hyaluronan binding proteins RHAMM, CD44 and link protein. EMBO J. 13, 286296.

(70) Chai, C., and Leong, K. W. (2007) Biomaterials approach to expand and direct differentiation of stem cells. Mol. Ther. 15, 467-480.

(71) Mallanna, S. K., and Rizzino, A. (2012) Systems biology provides new insights into the molecular mechanisms that control the fate of embryonic stem cells. J. Cell. Physiol. 227, 27-34.

(72) da Silva Meirelles, L., Fontes, A. M., Covas, D. T., and Caplan, A. I. (2009) Mechanisms involved in the therapeutic properties of mesenchymal stem cells. Cytokine Growth Factor Rev. 20, 419-427.

(73) Oh, I. H., and Humphries, R. K. (2012) Concise review: Multidimensional regulation of the hematopoietic stem cell state. Stem Cells 30, 82-88.

(74) Balian, G., Beck, G., Madhu, V., Sikes, R., Cui, Q., Liang, H., and Bush, J. (2010) Peptides from phage display library modulate gene expression in mesenchymal cells and potentiate osteogenesis in unicortical bone defects. J. Visualized Exp., DOI: 10.3791/2362.

(75) Cao, F. Y., Yin, W. N., Fan, J. X., Zhuo, R. X., and Zhang, X. Z. (2015) A novel function of BMHP1 and cBMHP1 peptides to induce the osteogenic differentiation of mesenchymal stem cells. Biomater. Sci. $3,345-351$.

(76) Cabanas-Danes, J., Nicosia, C., Landman, E., Karperien, M., Huskens, J., and Jonkheijm, P. (2013) A fluorogenic monolayer to detect the co-immobilization of peptides that combine cartilage targeting and regeneration. J. Mater. Chem. B 1, 1903-1908.

(77) Li, L., Klim, J. R., Derda, R., Courtney, A. H., and Kiessling, L. L. (2011) Spatial control of cell fate using synthetic surfaces to potentiate TGF-beta signaling. Proc. Natl. Acad. Sci. U. S. A. 108, 11745-11750.

(78) Gungormus, M., Branco, M., Fong, H., Schneider, J. P., Tamerler, C., and Sarikaya, M. (2010) Self assembled bi-functional peptide hydrogels with biomineralization-directing peptides. Biomaterials 31, 7266-7274.

(79) Segvich, S. J., Smith, H. C., and Kohn, D. H. (2009) The adsorption of preferential binding peptides to apatite-based materials. Biomaterials 30, 1287-1298.

(80) Weiger, M. C., Park, J. J., Roy, M. D., Stafford, C. M., Karim, A., and Becker, M. L. (2010) Quantification of the binding affinity of a specific hydroxyapatite binding peptide. Biomaterials 31, 2955-2963.

(81) Jin, H. E., Jang, J., Chung, J., Lee, H. J., Wang, E., Lee, S. W., and Chung, W. J. (2015) Biomimetic Self-Templated Hierarchical Structures of Collagen-Like Peptide Amphiphiles. Nano Lett. 15, $7138-7145$.

(82) Cigognini, D., Silva, D., Paloppi, S., and Gelain, F. (2014) Evaluation of Mechanical Properties and Therapeutic Effect of
Injectable Self-Assembling Hydrogels for Spinal Cord Injury. J. Biomed. Nanotechnol. 10, 309.

(83) Gelain, F., Bottai, D., Vescovi, A., and Zhang, S. (2006) Designer self-assembling peptide nanofiber scaffolds for adult mouse neural stem cell 3-dimensional cultures. PLoS One 1, e119.

(84) Gelain, F., Cigognini, D., Caprini, A., Silva, D., Colleoni, B., Donega, M., Antonini, S., Cohen, B. E., and Vescovi, A. (2012) New bioactive motifs and their use in functionalized self-assembling peptides for NSC differentiation and neural tissue engineering. Nanoscale 4, 2946-2957.

(85) Gelain, F., Panseri, S., Antonini, S., Cunha, C., Donega, M., Lowery, J., Taraballi, F., Cerri, G., Montagna, M., Baldissera, F., and Vescovi, A. (2011) Transplantation of nanostructured composite scaffolds results in the regeneration of chronically injured spinal cords. ACS Nano 5, 227-236.

(86) Gelain, F., Silva, D., Caprini, A., Taraballi, F., Natalello, A., Villa, O., Nam, K. T., Zuckermann, R. N., Doglia, S. M., and Vescovi, A. (2011) BMHP1-derived self-assembling peptides: hierarchically assembled structures with self-healing propensity and potential for tissue engineering applications. ACS Nano 5, 1845-1859.

(87) Lee, S. S., Hsu, E. L., Mendoza, M., Ghodasra, J., Nickoli, M. S., Ashtekar, A., Polavarapu, M., Babu, J., Riaz, R. M., Nicolas, J. D., Nelson, D., Hashmi, S. Z., Kaltz, S. R., Earhart, J. S., Merk, B. R., McKee, J. S., Bairstow, S. F., Shah, R. N., Hsu, W. K., and Stupp, S. I. (2015) Gel scaffolds of BMP-2-binding peptide amphiphile nanofibers for spinal arthrodesis. Adv. Healthcare Mater. 4, 131-141.

(88) Lin, C. C., and Anseth, K. S. (2009) Controlling Affinity Binding with Peptide-Functionalized Poly(ethylene glycol) Hydrogels. Adv. Funct. Mater. 19, 2325.

(89) Shah, R. N., Shah, N. A., Del Rosario Lim, M. M., Hsieh, C., Nuber, G., and Stupp, S. I. (2010) Supramolecular design of selfassembling nanofibers for cartilage regeneration. Proc. Natl. Acad. Sci. U. S. A. 107, 3293-3298.

(90) Rasmussen, U. B., Schreiber, V., Schultz, H., Mischler, F., and Schughart, K. (2002) Tumor cell-targeting by phage-displayed peptides. Cancer Gene Ther. 9, 606-612.

(91) Svensen, N., Walton, J. G., and Bradley, M. (2012) Peptides for cell-selective drug delivery. Trends Pharmacol. Sci. 33, 186-192.

(92) Barry, M. A., Dower, W. J., and Johnston, S. A. (1996) Toward cell-targeting gene therapy vectors: selection of cell-binding peptides from random peptide-presenting phage libraries. Nat. Med. 2, 299-305.

(93) Santos, J. L., Pandita, D., Rodrigues, J., Pego, A. P., Granja, P. L., Balian, G., and Tomas, H. (2010) Receptor-mediated gene delivery using PAMAM dendrimers conjugated with peptides recognized by mesenchymal stem cells. Mol. Pharmaceutics 7, 763-774.

(94) Liang, C., Guo, B., Wu, H., Shao, N., Li, D., Liu, J., Dang, L., Wang, C., Li, H., Li, S., Lau, W. K., Cao, Y., Yang, Z., Lu, C., He, X., Au, D. W., Pan, X., Zhang, B. T., Lu, C., Zhang, H., Yue, K., Qian, A., Shang, P., Xu, J., Xiao, L., Bian, Z., Tan, W., Liang, Z., He, F., Zhang, L., Lu, A., and Zhang, G. (2015) Aptamer-functionalized lipid nanoparticles targeting osteoblasts as a novel RNA interference-based bone anabolic strategy. Nat. Med. 21, 288-294.

(95) Zhang, G., Guo, B., Wu, H., Tang, T., Zhang, B. T., Zheng, L., He, Y., Yang, Z., Pan, X., Chow, H., To, K., Li, Y., Li, D., Wang, X., Wang, Y., Lee, K., Hou, Z., Dong, N., Li, G., Leung, K., Hung, L., He, F., Zhang, L., and Qin, L. (2012) A delivery system targeting bone formation surfaces to facilitate RNAi-based anabolic therapy. Nat. Med. 18, 307-314.

(96) Naumova, A. V., Modo, M., Moore, A., Murry, C. E., and Frank, J. A. (2014) Clinical imaging in regenerative medicine. Nat. Biotechnol. 32, 804-818.

(97) Molek, P., Strukelj, B., and Bratkovic, T. (2011) Peptide phage display as a tool for drug discovery: targeting membrane receptors. Molecules 16, 857-887.

(98) Solis, M. A., Chen, Y. H., Wong, T. Y., Bittencourt, V. Z., Lin, Y. C., and Huang, L. L. (2012) Hyaluronan regulates cell behavior: a potential niche matrix for stem cells. Biochem. Res. Int. 2012, 346972.

(99) Volk, S. W., Iqbal, S. A., and Bayat, A. (2013) Interactions of the Extracellular Matrix and Progenitor Cells in Cutaneous Wound Healing. Advances in wound care 2, 261-272. 
(100) Gray, B. P., and Brown, K. C. (2014) Combinatorial peptide libraries: mining for cell-binding peptides. Chem. Rev. 114, 1020-1081. (101) Cao, L., Li, B., Yi, P., Zhang, H., Dai, J., Tan, B., and Deng, Z. (2014) The interplay of T1- and T2-relaxation on T1-weighted MRI of hMSCs induced by Gd-DOTA-peptides. Biomaterials 35, 4168-4174.

(102) Barrow, M., Taylor, A., Murray, P., Rosseinsky, M. J., and Adams, D. J. (2015) Design considerations for the synthesis of polymer coated iron oxide nanoparticles for stem cell labelling and tracking using MRI. Chem. Soc. Rev. 44, 6733-6748.

(103) Taylor, A., Wilson, K. M., Murray, P., Fernig, D. G., and Levy, R. (2012) Long-term tracking of cells using inorganic nanoparticles as contrast agents: are we there yet? Chem. Soc. Rev. 41, 2707-2717.

(104) Yea, K., Xie, J., Zhang, H., Zhang, W., and Lerner, R. A. (2015) Selection of multiple agonist antibodies from intracellular combinatorial libraries reveals that cellular receptors are functionally pleiotropic. Curr. Opin. Chem. Biol. 26, 1-7. 